\title{
Radial Moment Calculations of Coupled Electron-Photon Beams
}

\author{
Brian C. Franke \\ Simulation Technology Research Department \\ Sandia National Laboratories \\ Albuquerque, New Mexico 87185
}

Edward W. Larsen

Department of Nuclear Engineering and Radiological Sciences

University of Michigan

Ann Arbor, Michigan 48109

3 copies submitted

20 figures

0 tables

28 text pages, including cover page

Send correspondence to:

Brian C. Franke

Sandia National Laboratories

P.O. Box 5800, MS 1179

Albuquerque, NM 87185

phone: (505)845-7609

fax: (505)844-0092

email: bcfrank@sandia.gov 


\section{DISCLAIMER}

This report was prepared as an account of work sponsored by an agency of the United States Government. Neither the United States Government nor any agency thereof, nor any of their employees, make any warranty, express or implied, or assumes any legal liability or responsibility for the accuracy, completeness, or usefulness of any information, apparatus, product, or process disclosed, or represents that its use would not infringe privately owned rights. Reference herein to any specific commercial product, process, or service by trade name, trademark, manufacturer, or otherwise does not necessarily constitute or imply its endorsement, recommendation, or favoring by the United States Government or any agency thereof. The views and opinions of authors expressed herein do not necessarily state or reflect those of the United States Government or any agency thereof. 


\section{DISCLAIMER}

Portions of this document may be illegible in electronic image products. Images are produced from the best available original document. 


\begin{abstract}
We consider the steady-state transport of normally incident pencil beams of radiation in slabs of material. A method has been developed for determining the exact radial moments of 3-D beams of radiation as a function of depth into the slab, by solving systems of 1$D$ transport equations. We implement these radial moment equations in the ONEBFP discrete ordinates code and simulate energy-dependent, coupled electron-photon beams using CEPXS-generated cross sections. Modified $P_{N}$ synthetic acceleration is employed to speed up the iterative convergence of the 1-D charged particle calculations. For high-energy photon beams, a hybrid Monte Carlo/discrete ordinates method is examined. We demonstrate the efficiency of the calculations and make comparisons with 3-D Monte Carlo calculations. Thus, by solving 1-D transport equations, we obtain realistic multidimensional information concerning the broadening of electron-photon beams. This information is relevant to fields such as industrial radiography, medical imaging, radiation oncology, particle accelerators, and lasers.
\end{abstract}




\section{INTRODUCTION}

One-dimensional $S_{N}$ calculations have been shown to be accurate and efficient for electron transport problems. ${ }^{1-3}$ These calculations are made easier by treating the most forwardpeaked elastic scatters by a Fokker-Planck approximation. ${ }^{4}$ In addition, effective numerical methods have been developed for dealing with Fokker-Planck scattering ${ }^{5}$ and for accelerating electron transport calculations. ${ }^{6,7} \mathrm{~S}_{\mathrm{N}}$ calculations can also be employed for multidimensional electron transport problems. ${ }^{8-10}$ However, these codes are much less tenable for high-energy problems involving a singular source, such as a pencil beam.

The multidimensional "beam problem" has been examined rather extensively. The first mathematical exploration of the topic seems to have been made by Enrico Fermi in a lecture in 1940. In considering cosmic rays incident on the atmosphere, Fermi simplified the description of the beam to an analytically-solvable form by assuming a non-absorbing, homogeneous medium with no energy-dependence: a thin slab with highly forward-peaked scattering so that the beam remains collimated and backscatter is negligible. In 1941, Rossi and Griesen provided a written account of Fermi's work. ${ }^{11}$ In 1948, Eyges extended Fermi's description to include energy dependence with the continuous slowing down approximation, correlating energy loss with pathlength travelled. ${ }^{12}$ While this analytical solution is quite useful, it has shortcomings: it neglects large-angle scattering, the low-energy diffusion of particles, and the production of secondary particles such as bremsstrahlung photons.

The problem of radiation beams with highly forward-peaked scattering has received attention in the past couple of decades, as attempts have been made to more accurately model the spreading of the beams. An overview of efforts within the medical physics community has been provided by Jette. ${ }^{13}$ Within the past 5 years, this problem has been examined increasingly within the nuclear engineering community as well. ${ }^{14-19}$

In this paper, we demonstrate that exact multidimensional information concerning the spreading of 3-D high-energy radiation beams can be obtained by solving 1-D transport problems. Specifically, we derive 1-D transport equations for the exact radial moments of a beam of radiation, by taking "transverse" space-angle moments of the underlying 3-D transport equation. For a radiation beam described by three spatial, one energy, and two directional dimensions, we take moments to obtain equations with one spatial, one energy, 
and one directional dimension. These equations yield moments information for the radial dimension of the beam. We solve these equations using a 1-D $S_{N}$ code with realistic cross sections. We are able to overcome many of the drawbacks of the Fermi-Eyges treatment by directly solving the linear transport equations, but we are limited to acquiring radial moments in layered slab geometries. While our solutions are not analytical, the necessary 1-D calculations are fast and accurate.

The remainder of this paper is organized as follows. In Section II, we extend the radial moment equations previously derived by $\operatorname{Larsen}^{20}$ to coupled multiple-species, energy-dependent problems. In Section III, we discuss the methods and the computer codes employed to solve the energy-dependent transport problems that we examine and the numerical methods relevant to the implementation of the radial moment equations. In Section IV, we examine the efficiency of the modified $P_{\mathrm{N}}$ synthetic acceleration method for speeding up the iterative convergence of charged particle $S_{N}$ calculations. ${ }^{6}$ In Section V, radial moment results are presented for coupled electron-photon beam problems. We conclude with a brief summary of our work.

The work contained in this paper is taken from the $\mathrm{Ph}$.D. thesis of the first author (B.C.F.). ${ }^{21}$ We refer the readers of this paper to the thesis for additional details concerning theory, implementation and numerical results.

\section{Radial Moment Equations}

The slab-system $(0<z<Z)$ pencil beam radiation transport problem is described by 3-D transport equations coupled for each species of particle. We specifically consider the application of these equations to coupled electron-photon beam problems. However, these equations can easily be adapted to other coupled particle transport problems and to problems with arbitrary sources and boundary conditions. In the case of an electron-

photon beam with positrons, we have three coupled transport equations. In this paper, we denote different species of particles with superscripts 1,2 , and 3 for photons, electrons, and positrons, respectively.

To model the highly forward-peaked scattering of electrons and positrons, the most forward-peaked interactions are treated by the Fokker-Planck approximation. This treat- 
ment is a precursor to the implementation of the $S_{N}$ method, for which the accuracy of the scattering cross section is limited by the order of the moments expansion. Those scattering interactions that are too forward-peaked to be accurately modelled by $S_{N}$ are treated by the Fokker-Planck approximation. Thus, the distributions of photons, electrons, and positrons are described by a system of coupled Boltzmann-Fokker-Planck equations: ${ }^{4}$

$$
\begin{aligned}
& -\frac{\partial}{\partial E} S^{(k)}(z, E) \psi^{(k)}(\underline{r}, \underline{\Omega}, E)+\underline{\Omega} \cdot \underline{\nabla} \psi^{(k)}(\underline{r}, \underline{\Omega}, E)+\Sigma_{t}^{(k)}(z, E) \psi^{(k)}(\underline{r}, \underline{\Omega}, E) \\
& \quad=\frac{\alpha^{(k)}(z, E)}{2}\left\{\frac{\partial}{\partial \mu}\left(1-\mu^{2}\right) \frac{\partial}{\partial \mu}+\frac{1}{\left(1-\mu^{2}\right)} \frac{\partial^{2}}{\partial \phi^{2}}\right\} \psi^{(k)}(\underline{r}, \underline{\Omega}, E) \\
& \quad+\sum_{j=1}^{3} \int_{4 \pi} \int_{0}^{E_{0}} \Sigma^{j \rightarrow k}\left(z, \underline{\Omega^{\prime}} \cdot \underline{\Omega}, E^{\prime} \rightarrow E\right) \psi^{(j)}\left(\underline{r}, \underline{\Omega}^{\prime}, E^{\prime}\right) d \Omega^{\prime} d E^{\prime}, k=1,2,3 .
\end{aligned}
$$

In these equations,,$\Sigma^{j \rightarrow k}$ are cross sections for the production of species $k$ from species $j$. These cross sections include scattering as well as secondary production. $\Sigma^{2 \rightarrow 3}$, the production of positrons by electrons, is the only cross section that is always zero. In the case of photons, which do not employ the Fokker-Planck approximation, the stopping power $S$ and the momentum transfer coefficient $\alpha$ are zero. The slab-system is radially homogeneous but can be axially heterogeneous ("layered" in the $z$-direction).

In general, we are interested in a beam of photons, electrons, and/or positrons incident on the left edge of the slab. For such a problem, the boundary conditions are:

$$
\begin{array}{cc}
\psi^{(k)}(x, y, 0, \mu, \phi, E)=\xi^{(k)}(x, y, \mu, \phi, E), & 0<\mu \leq 1, \quad k=1,2,3, \\
\psi^{(k)}(x, y, Z, \mu, \phi, E)=0, & -1 \leq \mu<0, \quad k=1,2,3,
\end{array}
$$

where $\xi^{(k)}$ are prescribed.

The "first" equations that we solve are obtained by "transversely" integrating the 3-D transport equations over $x, y$, and azimuthal angle, $\phi$. This yields the following exact 1-D equations for the transversely-integrated angular flux for each species: 


$$
\begin{gathered}
-\frac{\partial}{\partial E} S^{(k)}(z, E) \psi_{0}^{(k)}(z, \mu, E)+\mu \frac{\partial}{\partial z} \psi_{0}^{(k)}(z, \mu, E)+\Sigma_{t}^{(k)}(z, E) \psi_{0}^{(k)}(z, \mu, E) \\
=\frac{\alpha^{(k)}(z, E)}{2} \frac{\partial}{\partial \mu}\left(1-\mu^{2}\right) \frac{\partial}{\partial \mu} \psi_{0}^{(k)}(z, \mu, E) \\
+\sum_{j=1}^{3} \sum_{n=0}^{\infty}(2 n+1) P_{n}(\mu) \int_{0}^{E_{0}} \Sigma_{n}^{j+k}\left(z, E^{\prime} \rightarrow E\right) \phi_{n, 0}^{(j)}\left(z, E^{\prime}\right) d E^{\prime}
\end{gathered}
$$

with boundary conditions:

$$
\begin{array}{cc}
\psi_{0}^{(k)}(0, \mu, E)=\xi_{0}^{(k)}(\mu, E), & 0<\mu \leq 1 \\
\psi_{0}^{(k)}(Z, \mu, E)=0, & -1 \leq \mu<0
\end{array}
$$

Here we have defined:

$$
\begin{gathered}
\psi_{0}^{(k)}(z, \mu, E) \equiv \int_{0}^{2 \pi} \int_{-\infty}^{\infty} \int_{-\infty}^{\infty} \psi^{(k)}(\underline{r}, \underline{\Omega}, E) d x d y d \phi \\
\phi_{n, 0}^{(k)}(z, E) \equiv \frac{1}{2} \int_{-1}^{1} P_{n}\left(\mu^{\prime}\right) \psi_{0}^{(k)}\left(z, \mu^{\prime}, E\right) d \mu^{\prime} \\
\xi_{0}^{(k)}(\mu, E) \equiv \int_{0}^{2 \pi} \int_{-\infty}^{\infty} \int_{-\infty}^{\infty} \xi^{(k)}(x, y, \mu, \phi, E) d x d y d \phi
\end{gathered}
$$

The "second" equations that we solve are obtained by transversely integrating the 3-D transport equations multiplied by $(x \cos \phi+y \sin \phi)$. This yields the following exact 1-D equations for the first radial moment of the net radial current:

$$
\begin{aligned}
& -\frac{\partial}{\partial E} S^{(k)}(z, E) \psi_{1}^{(k)}(z, \mu, E)+\mu \frac{\partial}{\partial z} \psi_{1}^{(k)}(z, \mu, E)+\Sigma_{t}^{(k)}(z, E) \psi_{1}^{(k)}(z, \mu, E) \\
& \quad=\sqrt{1-\mu^{2}} \psi_{0}^{(k)}(z, \mu, E)+\frac{\alpha^{(k)}(z, E)}{2}\left\{\frac{\partial}{\partial \mu}\left(1-\mu^{2}\right) \frac{\partial}{\partial \mu}-\frac{1}{\left(1-\mu^{2}\right)}\right\} \psi_{1}^{(k)}(z, \mu, E) \\
& \quad+\sum_{j=1}^{3} \sum_{n=1}^{\infty} \frac{2 n+1}{n(n+1)} P_{n, 1}(\mu) \int_{0}^{E_{0}} \Sigma_{n}^{j \rightarrow k}\left(z, E^{\prime} \rightarrow E\right) \phi_{n, 1}^{(j)}\left(z, E^{\prime}\right) d E^{\prime}
\end{aligned}
$$

with boundary conditions:

$$
\begin{array}{cc}
\psi_{0}^{(k)}(0, \mu, E)=\xi_{1}^{(k)}(\mu, E), & 0<\mu \leq 1 \\
\psi_{0}^{(k)}(Z, \mu, E)=0, & -1 \leq \mu<0
\end{array}
$$


Here we have defined:

$$
\begin{gathered}
\psi_{1}^{(k)}(z, \mu, E) \equiv \int_{0}^{2 \pi} \int_{-\infty}^{\infty} \int_{-\infty}^{\infty}(x \cos \phi+y \sin \phi) \psi^{(k)}(\underline{r}, \underline{\Omega}, E) d x d y d \phi \\
\phi_{n, 1}^{(k)}(z, E) \equiv \frac{1}{2} \int_{-1}^{1} P_{n, 1}\left(\mu^{\prime}\right) \psi_{1}^{(k)}\left(z, \mu^{\prime}, E\right) d \mu^{\prime} \\
\xi_{1}^{(k)}(\mu, E) \equiv \int_{0}^{2 \pi} \int_{-\infty}^{\infty} \int_{-\infty}^{\infty}(x \cos \phi+y \sin \phi) \xi^{(k)}(x, y, \mu, \phi, E) d x d y d \phi
\end{gathered}
$$

Eqs. (10) have source terms containing the transversely integrated flux $\psi_{0}^{(k)}(z, \mu, E)$ determined by solving Eqs. (4). They also have a modified Fokker-Planck term and a scattering cross section expansion based on the first-order associated Legendre functions, $P_{n, 1}(\mu)$.

The "third" equations that we solve are obtained by transversely integrating the 3-D transport equations multiplied by $\left(x^{2}+y^{2}\right)$. This yields the following exact 1-D equations for the second radial moment of the flux:

$$
\begin{gathered}
-\frac{\partial}{\partial E} S^{(k)}(z, E) \psi_{2}^{(k)}(z, \mu, E)+\mu \frac{\partial}{\partial z} \psi_{2}^{(k)}(z, \mu, E)+\Sigma_{t}^{(k)}(z, E) \psi_{2}^{(k)}(z, \mu, E) \\
=\sqrt{1-\mu^{2}} \psi_{1}^{(k)}(z, \mu, E)+\frac{\alpha^{(k)}(z, E)}{2} \frac{\partial}{\partial \mu}\left(1-\mu^{2}\right) \frac{\partial}{\partial \mu} \psi_{2}^{(k)}(z, \mu, E) \\
\quad+\sum_{j=1}^{3} \sum_{n=0}^{\infty}(2 n+1) P_{n}(\mu) \int_{0}^{E_{0}} \Sigma_{n}^{j \rightarrow k}\left(z, E^{\prime} \rightarrow E\right) \phi_{n, 2}^{(j)}\left(z, E^{\prime}\right) d E^{\prime},
\end{gathered}
$$

with boundary conditions:

$$
\begin{array}{cc}
\psi_{0}^{(k)}(0, \mu, E)=\xi_{2}^{(k)}(\mu, E), & 0<\mu \leq 1, \\
\psi_{0}^{(k)}(Z, \mu, E)=0, & -1 \leq \mu<0 .
\end{array}
$$

Here we have defined:

$$
\begin{gathered}
\psi_{2}^{(k)}(z, \mu, E) \equiv \int_{0}^{2 \pi} \int_{-\infty}^{\infty} \int_{-\infty}^{\infty}\left(x^{2}+y^{2}\right) \psi^{(k)}(\underline{r}, \underline{\Omega}, E) d x d y d \phi \\
\phi_{n, 2}^{(k)}(z, E) \equiv \frac{1}{2} \int_{-1}^{1} P_{n}\left(\mu^{\prime}\right) \psi_{2}^{(k)}\left(z, \mu^{\prime}, E\right) d \mu^{\prime} \\
\xi_{2}^{(k)}(\mu, E) \equiv \int_{0}^{2 \pi} \int_{-\infty}^{\infty} \int_{-\infty}^{\infty}\left(x^{2}+y^{2}\right) \xi^{(k)}(x, y, \mu, \phi, E) d x d y d \phi
\end{gathered}
$$

Eqs. (16) contain source terms based on the first radial moment of the net radial current 
$\psi_{1}^{(k)}(z, \mu, E)$ determined by solving Eqs. (10).

These three sets of coupled equations, which have been derived from the 3-D equations without approximation, can be solved sequentially: first Eqs. (4), then Eqs. (10) and then Eqs. (16). If the Fokker-Planck approximation is not relied upon, these equations are exact. They yield the zeroth and second radial moments of the photon, electron, and positron angular flux as a function of depth and energy. The above derivation can be extended to higher-order radial moments as well.

\section{Numerical Methods}

The information that is generally of the most interest in charged particle transport problems is energy deposition or dose. The transverse energy deposition is:

$$
\begin{aligned}
\hat{D}(z) & \equiv \int_{0}^{E_{0}} \int_{4 \pi} \int_{0}^{\infty}\left[\sum_{k=1}^{3} \Sigma_{e}^{(k)}(z, E) \psi^{(k)}(\underline{r}, \underline{\Omega}, E)\right] r d r d \Omega d E \\
& =\int_{0}^{E_{0}}\left[\sum_{k=1}^{3} \Sigma_{e}^{(k)}(z, E) \phi_{0,0}^{(k)}(z, E)\right] d E
\end{aligned}
$$

where $\Sigma_{e}$ is an energy deposition cross section. [Only charged particles deposit dose. However, in the context of the calculation, photons can deposit dose by scattering below the numerical cutoff energy or by producing secondary particles below the cutoff energy.] The rms radius of the energy deposition is:

$$
\begin{aligned}
\hat{r}(z) & \equiv\left\{\frac{\int_{0}^{E_{0}} \int_{4 \pi} \int_{0}^{\infty} r^{2}\left[\sum_{k=1}^{3} \Sigma_{e}^{(k)}(z, E) \psi^{(k)}(\underline{r}, \underline{\Omega}, E)\right] r d r d \Omega d E}{\int_{0}^{E_{0}} \int_{4 \pi} \int_{0}^{\infty}\left[\sum_{k=1}^{3} \Sigma_{e}^{(k)}(z, E) \psi^{(k)}(\underline{r}, \underline{\Omega}, E)\right] r d r d \Omega d E}\right\}^{\frac{1}{2}} \\
& =\left\{\frac{\int_{0}^{E_{0}}\left[\sum_{k=1}^{3} \Sigma_{e}^{(k)}(z, E) \phi_{0,2}^{(k)}(z, E)\right] d E}{\hat{D}(z)}\right\}
\end{aligned}
$$

We see that $\hat{D}(z)$ provides the total energy deposition at depth $z$. Conceptually, this quantity is the standard output of a conventional 1-D particle transport code. However, $\hat{r}(z)$ provides 
the exact rms radius of the energy deposition at depth $z$. This is a true multidimensional quantity that has not previously been calculable by $1-D$ codes.

We evaluate the efficiency of this method by comparing the accuracy of calculations as a function of computation time. We have calculated $\hat{D}(z)$ and $\hat{r}(z)$ for several radiation beams. We desire an accurate calculation across the entire depth of the slab, so we define accuracy in terms of an $\mathrm{L}_{2}$-norm of the relative difference between results as a function of depth:

$$
\epsilon=\left[\frac{1}{Z} \int_{0}^{Z}\left(\frac{\hat{r}_{b}(z)-\hat{r}(z)}{\hat{r}_{b}(z)}\right)^{2} d z\right]^{\frac{1}{2}}
$$

where $\epsilon$ is the relative error, and $\hat{r}_{b}$ is the benchmark calculation to which other calculations are compared.

The benchmarks are established by high-order calculations with the $\mathrm{S}_{\mathrm{N}}$ and Monte Carlo methods. Because the $S_{N}$ and Monte Carlo codes use different cross sections to solve the transport problems, the two types of codes will converge toward slightly different results as discretization and statistical errors are reduced. For this reason, $\mathrm{S}_{\mathrm{N}}$ calculations are compared to an $\mathrm{S}_{\mathrm{N}}$ benchmark calculation, and Monte Carlo calculations are compared to a Monte Carlo benchmark calculation. The $\mathrm{S}_{\mathrm{N}}$ and Monte Carlo benchmark calculations are compared to each other, to check for approximate agreement between the two methods.

Computational timings do not include the time required for cross section generation, either with XGEN for use in CYLTRAN or with CEPXS for use in ONEBFP. All timings were performed on a single $533 \mathrm{MHz}$ processor on the "Tera" computing cluster at Lawrence Livermore National Laboratory. ${ }^{22}$

\section{III.A. Discrete Ordinates}

We have implemented our radial moments method in the ONEBFP code developed at Los Alamos National Laboratory. ${ }^{23}$ This 1-D multigroup $S_{N}$ code solves the Boltzmann-FokkerPlanck equation for charged particles and the Boltzmann equation for neutral particles. It uses a quadratic discontinuous discretization scheme in space and energy for transport calculations ${ }^{24}$ and employs $\mathrm{S}_{2}$ acceleration of the scalar flux and current with linear discontinuous 
discretization. ${ }^{25}$ It also employs a Fokker-Planck angular differencing scheme in the transport calculation. ${ }^{5} \mathrm{~S}_{\mathrm{N}}$ with Gauss-Legendre quadrature is used for the angular discretization.

We utilize cross section moments generated by the CEPXS code developed at Sandia National Laboratories. ${ }^{26}$ This code models the physics of photon, electron, and positron interactions ${ }^{27}$ and has been validated against results from other codes and experimental data. $^{3}$

Alterations were made to ONEBFP to implement the radial moment equations derived above, and to improve the efficiency with which these equations are solved. The code was adapted to utilize first-order Legendre functions to solve the "second" equations [(10)-(12)]. In addition, an $S_{2}$ acceleration scheme was implemented for these equations. Then, the $S_{2}$ acceleration schemes for all equations were generalized to modified $P_{N}$ synthetic acceleration to allow for the acceleration of higher-order angular moments.

\section{III.B. Monte Carlo}

The radial moment results calculated with ONEBFP are compared with results from the CYLTRAN Monte Carlo code that is part of Version 3.0 of the Integrated TIGER Series (ITS) codes developed at Sandia National Laboratories. ${ }^{28}$ CYLTRAN was chosen because of its close relationship with CEPXS in terms of the physics modelled.

\section{III.C. Hybrid Monte Carlo/Discrete Ordinates}

In the case of high-energy photon beams, it is advantageous to employ a hybrid Monte Carlo/ $\mathrm{S}_{\mathrm{N}}$ method. Like electrons, high-energy photons undergo highly forward-peaked scattering. Unlike electrons, the photons do not use a Fokker-Planck approximation. Thus, a very high-order $S_{N}$ cross section moment expansion is required to accurately represent the angular scattering distribution. Unfortunately, it is computationally expensive to use CEPXS to generate high-order expansions of photon cross section moments. The hybrid method avoids this difficulty by using a modified version of the CYLTRAN code to simulate the incident photons through their lives. We record the spatial and angular distribution of all secondary electrons and positrons produced and use this as a source in ONEBFP. All 
subsequent transport calculations are performed with ONEBFP, including bremsstrahlung and annihilation photon transport. The advantage of this method is that CYLTRAN can efficiently and exactly simulate the nearly singular primary photon transport. The ONEBFP portion of the calculation requires a relatively low-order quadrature, because it begins with a distributed source of charged particles in each of the radial moment equations.

\section{ACCELERATION}

The Diffusion Synthetic Acceleration (DSA) method effectively accelerates transport problems with weakly anisotropic scattering by correcting the two lowest angular moments. ${ }^{29}$ The Modified $\mathrm{P}_{\mathrm{N}}$ Synthetic Acceleration (MPSA) method is a generalization of DSA that effectively accelerates calculations involving highly anisotropic scattering by correcting higherorder angular moments. ${ }^{6}$ For $n$ even, the MPSA method uses the following equations to accelerate the convergence of the $n$-th and $(n+1)$-th angular moments of the "first" equations $[(4)-(6)]$ :

$$
\begin{aligned}
& -S^{(k)}(z) \frac{\partial}{\partial E} f_{n}^{(k)}(z, E)+\frac{n+1}{2 n+1} \frac{\partial}{\partial z} f_{n+1}^{(k)}(z, E) \\
& +\Sigma_{t}^{(k)}(z) f_{n}^{(k)}(z, E)-\Sigma_{n}^{k \rightarrow k}(z) f_{n}^{(k)}(z, E)+\frac{\alpha^{(k)}(z)}{2} n(n+1) f_{n}^{(k)}(z, E) \\
& =\Sigma_{n}^{k \rightarrow k}(z) g_{n}^{(k)}(z, E)-\frac{\alpha^{(k)}(z)}{2} n(n+1) g_{n}^{(k)}(z, E) \\
& -S^{(k)}(z) \frac{\partial}{\partial E} f_{n+1}^{(k)}(z, E)+\frac{n+1}{2 n+3} \frac{\partial}{\partial z} f_{n}^{(k)}(z, E) \\
& +\Sigma_{t}^{(k)}(z) f_{n+1}^{(k)}(z, E)-\Sigma_{n+1}^{k \rightarrow k}(z) f_{n+1}^{(k)}(z, E)+\frac{\alpha^{(k)}(z)}{2}(n+1)(n+2) f_{n+1}^{(k)}(z, E) \\
& =\Sigma_{n+1}^{k \rightarrow k}(z) g_{n+1}^{(k)}(z, E)-\frac{\alpha^{(k)}(z)}{2}(n+1)(n+2) g_{n+1}^{(k)}(z, E) .
\end{aligned}
$$

Here $f_{n}^{(k)}$ and $g_{n}^{(k)}$ are the correction and residual terms for the $n$-th Legendre moment of the flux. [Eqs. (25) and (26) are obtained by taking the $n$-th and $(n+1)$-th Legendre moments of the exact 1-D equations for the transport correction and discarding terms involving the $(n-1)$-th and $(n+2)$-th angular moments of the correction. This discarding of certain terms is a partial decoupling that somewhat reduces the efficiency of these acceleration equations, 
but it makes them much easier to solve.] The boundary conditions for Eqs. (25) and (26) are, for $n$ even:

$$
f_{n}^{(k)}(0, E)=-\delta_{n, 0} 3 \bar{\mu}_{0} f_{1}^{(k)}(0, E), \quad f_{n}^{(k)}(Z, E)=\delta_{n, 0} 3 \bar{\mu}_{0} f_{1}^{(k)}(Z, E)
$$

where

$$
\bar{\mu}_{0}=\left[\sum_{m=1}^{N} \mu_{m}^{2} w_{m}\right] /\left[\sum_{m=1}^{N}\left|\mu_{m}\right| w_{m}\right]
$$

Eqs. (25) and (26) are independent of the acceleration of other moments and other particle species. For $n=0$, these equations become the $P_{1}$ acceleration equations of the DSA method.

We have derived similar acceleration equations for the "second" equations [(10)-(12)] based on the first-order Legendre functions. For $n$ odd, the MPSA method uses the following equations to accelerate the $n$-th and $(n+1)$-th first-order Legendre moments of the flux:

$$
\begin{aligned}
& -S^{(k)}(z) \frac{\partial}{\partial E} f_{n}^{(k)}(z, E)+\frac{n}{2 n+1} \frac{\partial}{\partial z} f_{n+1}^{(k)}(z, E) \\
& +\Sigma_{t}^{(k)}(z) f_{n}^{(k)}(z, E)-\Sigma_{n}^{k \rightarrow k}(z) f_{n}^{(k)}(z, E)+\frac{\alpha^{(k)}(z)}{2} n(n+1) f_{n}^{(k)}(z, E) \\
& =\Sigma_{n}^{k \rightarrow k}(z) g_{n}^{(k)}(z, E)-\frac{\alpha^{(k)}(z)}{2} n(n+1) g_{n}^{(k)}(z, E), \\
& -S^{(k)}(z) \frac{\partial}{\partial E} f_{n+1}^{(k)}(z, E)+\frac{n+2}{2 n+3} \frac{\partial}{\partial z} f_{n}^{(k)}(z ; E) \\
& +\Sigma_{t}^{(k)}(z) f_{n+1}^{(k)}(z, E)-\Sigma_{n+1}^{k \rightarrow k}(z) f_{n+1}^{(k)}(z, E)+\frac{\alpha^{(k)}(z)}{2}(n+1)(n+2) f_{n+1}^{(k)}(z, E) \\
& =\Sigma_{n+1}^{k \rightarrow k}(z) g_{n+1}^{(k)}(z, E)-\frac{\alpha^{(k)}(z)}{2}(n+1)(n+2) g_{n+1}^{(k)}(z, E) .
\end{aligned}
$$

Eqs. (29) and (30) are obtained by taking the $n$-th and $(n+1)$-th first-order Legendre moments of the exact 1-D equations for the transport correction to Eqs. (10) and discarding terms involving the $(n-1)$-th and $(n+2)$-th first-order Legendre moments of the correction. The above equations have not been previously derived. However, the derivation is closely 
analogous to the derivation of Eqs. (25) and (26). The boundary conditions are, for $n$ odd:

$$
f_{n}^{(k)}(0, E)=-\delta_{n, 1} \frac{5}{3} \bar{\mu}_{1} f_{2}^{(k)}(0, E), \quad f_{n}^{(k)}(Z, E)=\delta_{n, 1} \frac{5}{3} \bar{\mu}_{1} f_{2}^{(k)}(Z, E),
$$

where

$$
\bar{\mu}_{1}=\left[\sum_{m=1}^{N}\left(1-\mu_{m}^{2}\right) \mu_{m}^{2} w_{m}\right] /\left[\sum_{m=1}^{N}\left(1-\mu_{m}^{2}\right)\left|\mu_{m}\right| w_{m}\right]
$$

The "third" equations [(16)-(18)] are based on the usual Legendre polynomials and use acceleration equations of the form of Eqs. (25) and (26). Here, as in the other transport equations, one obtains the acceleration equations for photons by setting the stopping power $S$ and the momentum transfer coefficient $\alpha$ to zero.

\section{RESULTS}

\section{V.A. Electron Beam}

We consider a monoenergetic $10 \mathrm{MeV}$ electron pencil beam normally incident at zero radius on the left side of a $0.5 \mathrm{~cm}$ copper slab. Fig. 1 shows the transversely integrated energy deposition as a function of depth, $\hat{D}(z)$, and illustrates that the width of the slab is almost the $10 \mathrm{MeV}$ electron range. A relative difference of $1.561 \times 10^{-2} \pm 4 \times 10^{-5}$ exists between the ONEBFP and CYLTRAN calculations shown here. These differences exist because of discretization and statistical errors, but also due to differences in the cross sections and methods used. Therefore, slight differences in the results of the two methods persist, even as higher-order calculations are used.

Fig. 2 shows the rms radius of the energy deposition as a function of depth, $\hat{r}(z)$, and illustrates the broadening of this beam. We note that in this and all other problems, the beam does not have $\hat{r}(0)=0$, due to electrons that turn around and leak out of the incident edge of the slab. A relative difference of $2.15 \times 10^{-2} \pm 3 \times 10^{-4}$ exists between these two calculations.

The results shown in Figs. 1 and 2 are used as the "benchmarks" for computing the relative error in less accurate results obtained with lower-order calculations of each method. 
CYLTRAN results are compared with the CYLTRAN benchmark, obtained using $10^{8}$ incident electrons. ONEBFP results are compared with the ONEBFP benchmark, obtained using 100 energy groups, the 128 angle Gauss-Legendre quadrature set, and 25 spatial cells.

In Fig. 3, we show the relative error versus computation time of low-order CYLTRAN and ONEBFP calculations of the energy deposition compared with their respective benchmark calculations. In Fig: 4, we show the relative errors in the rms radius of the energy deposition for the same calculations. For calculating the rms radius in this problem, we find that the $\mathrm{S}_{\mathrm{N}}$ code is more than one order of magnitude faster than the Monte Carlo code. CYLTRAN requires approximately $300 \mathrm{~s}$ to achieve a $5 \%$ relative error and $15,000 \mathrm{~s}$ to achieve a $1 \%$ relative error, while ONEBFP requires $25 \mathrm{~s}$ and $1,200 \mathrm{~s}$, respectively. In these error versus time plots, the CYLTRAN timings were taken with increasing numbers of incident electrons. ONEBFP timings were taken with 25 spatial cells, and a series of results are shown with quadrature orders ranging from 8 up to 128 for both 25 and 100 logarithmic energy groups from $10 \mathrm{MeV}$ to $0.01 \mathrm{MeV}$. Two other series of results are shown for 16 and 64 order quadratures with $15,25,50,75$, and 100 logarithmic energy groups. All of the ONEBFP calculations shown here used four-moment MPSA.

It is important to emphasize that the relative errors shown in Figs. 3 and 4 are based on comparisons with high-order calculations, not with experimental benchmarks. Even the highorder calculations rely on approximate numerical modeling of the true physics. In general, the calculated energy deposition values differ from accurate experimental measurements by perhaps a few percent. Also, differences in the numerical methods employed (due mostly to different cross sections) result in minor discrepencies between the Monte Carlo and $S_{N}$ benchmarks. Therefore, in examining the efficiency of calculations, one may usually consider relative errors below a few percent to be insignificant.

\section{V.A.1. Modified $P_{N}$ Synthetic Acceleration}

The MPSA equations were implemented in ONEBFP using linear discontinuous discretization in space and energy. MPSA yields the possibility of optimizing the number of flux moments to be accelerated. However, the optimum number of accelerated moments depends upon a number of factors, such as the spectral radius of the calculation, the rela- 
tive speeds of the $\mathrm{S}_{\mathrm{N}}$ and MPSA calculations, and the anisotropy of scattering and the flux distribution. These effects are illustrated in Figs. 5 and 6. Fig. 5 shows the convergence of an $\mathrm{S}_{32}$ calculation for a single energy group in which the flux is mildly anisotropic with varying levels of MPSA. Fig. 6 shows the convergence of a similar calculation for a single energy group in which the flux is stongly anisotropic.

The effectiveness of the acceleration method for the $10 \mathrm{MeV}$ electron problem is shown in Figs. 7 and 8. Results are shown for ONEBFP calculations, varying the quadrature order from 8 to 128 with 25 and 100 energy groups using no acceleration, $S_{2}$ acceleration, and four-moment MPSA. We find that $S_{2}$ acceleration speeds up calculations by approximately a factor of 2, and four-moment MPSA speeds up the calculations by approximately a factor of 2 beyond $S_{2}$ acceleration.

In some cases, MPSA was found to be unstable. This occurred when optically thick spatial cells and a course energy group structure were used. We believe that this instability is due to the linear discontinuous discretization employed. These instabilities are not predicted by the infinite medium Fourier analysis, and were more likely to occur when accelerating more moments with MPSA. Four-moment MPSA was generally (but not always) stable, while $S_{2}$ acceleration was stable in all physical cases examined.

\section{V.B. Photon Beam}

Next, we consider a monoenergetic $50 \mathrm{MeV}$ photon beam normally incident at zero radius on a $0.5 \mathrm{~cm}$ copper slab with vacuum boundary conditions. Figs. 9 and 10 show $\hat{D}(z)$ and $\hat{r}(z)$, respectively. Fig. 10 shows that the distribution of energy deposition narrows with depth rather than broadens. While the incident beam is narrow, energy is deposited by charged particles rather than photons. The electrons and positrons at the incident edge of the slab are due to backscattered particles, and therefore have a relatively broad distribution. As illustrated in Fig. 9, the slab is sufficiently thin that the beam does not achieve electronic equilibrium, relatively few particles are backscattered, and little energy is deposited at the incident edge of the slab.

In this case, the relative differences in the energy deposition distributions between the $\mathrm{S}_{\mathrm{N}}$ and hybrid benchmarks and the Monte Carlo benchmark are $5.75 \times 10^{-3} \pm 6 \times 10^{-5}$ and 
$3.9 \times 10^{-3} \pm 1 \times 10^{-4}$. The relative differences in the rms radius of the energy deposition are $3.05 \times 10^{-2} \pm 4 \times 10^{-4}$ and $3.42 \times 10^{-2} \pm 4 \times 10^{-4}$, respectively. The CYLTRAN benchmark was obtained using $10^{9}$ photon histories and forced photon collisions. The ONEBFP benchmark was obtained using 25 spatial cells, 100 energy groups, and a 128 angle quadrature. The hybrid benchmark was obtained using $10^{9}$ photon histories in the Monte Carlo portion of the calculation with forced photon collisions and using the same parameters for the $S_{N}$ portion of the calculation as were used for the ONEBFP benchmark.

In Fig. 11, we show the relative error in $\hat{D}(z)$ for the Monte Carlo, $\mathrm{S}_{\mathrm{N}}$, and hybrid methods for the $50 \mathrm{MeV}$ problem. Likewise, we show the relative error in $\hat{r}(z)$ in Fig. 12. The Monte Carlo portion of the hybrid calculations was performed with $10^{6}$ photon histories for the 25 energy group calculations. For the 100 energy group calculations, we used $10^{6}$ photon histories for the first three points (corresponding to 8, 16, and 24 order quadrature in the $S_{N}$ portion of the calculation) and $10^{7}$ photon histories for the remaining points. The $S_{N}$ calculations and $S_{N}$ portion of the hybrid calculations used 25 spatial cells with increasing quadrature orders for 25 and 100 energy groups. We employed four-moment MPSA in all cases except for the 25 energy group cases with 96 and 128 quadrature orders. In these two ONEBFP and hybrid calculations, four-moment MPSA was unstable and $\mathrm{S}_{2}$ acceleration was used.

ONEBFP performs poorly with low-order quadratures in this problem, but the hybrid method performs well. This is because the high-energy incident photons undergo highly foward-peaked scattering interactions. While ONEBFP uses the Fokker-Planck approximation to model the highly forward-peaked scattering of electrons, no such approximation is made for the photons. Thus, the ONEBFP cross section moments expansion and angular quadrature must be sufficient to accurately model the highly anisotropic scattering and resulting angular photon distribution. This is demonstrated in Figs. 11 and 12 by the strong dependence of the accuracy of ONEBFP calculations on the quadrature order.

Figs. 11 and 12 show that to achieve a $5 \%$ relative error in the rms radius calculation, the hybrid, Monte Carlo, and $S_{N}$ methods require approximately $40 \mathrm{~s}, 700 \mathrm{~s}$, and 5,000 s, respectively. To achieve a $1 \%$ relative error, the hybrid method requires approximately 2,000 $\mathrm{s}$, while the Monte Carlo method requires $12,000 \mathrm{~s}$. 
The hybrid method is accurate because the incident photon interactions are exactly modelled. Bremsstrahlung photons are the highest-energy photons that are simulated in the $S_{N}$ portion of the hybrid method. A low-order quadrature is sufficient here, because these photons have a less anisotropic angular distribution. An alternative to the hybrid method is to employ high-order quadratures and cross section moment expansions for primary photons in ONEBFP and low-order quadratures for the subsequent particle transport. This alternative is less appealing, due to the high cost of calculating photon cross section moments in CEPXS. It is more efficient to extract the incident photon transport information from CYLTRAN.

\section{V.C. Thin Slabs}

We have found that the highly anisotropic distribution of the incident photon beam can be conveniently modelled using the hybrid method. However, it is also possible for a highly anisotropic distribution of secondary photons to occur as a result of leakage from a thin slab. The ability to use a higher order angular quadrature for the photon transport calculation than for the charged particle transport calculation is an alteration we made to ONEBFP that allows the radial moments to be accurately calculated in thin slabs. To examine such a case, we consider a monoenergetic $10 \mathrm{MeV}$ photon beam normally incident at zero radius on a $0.1 \mathrm{~cm}$ copper slab with vacuum boundary conditions. The energy deposition as a function of depth is plotted in Fig. 13, and the rms radius of the energy deposition is plotted in Fig. 14.

The relative difference in the energy deposition distributions between the hybrid benchmark and the Monte Carlo benchmark is $4.76 \times 10^{-3} \pm 8 \times 10^{-5}$. The relative difference in the rms radius of the energy deposition is $3.2 \times 10^{-2} \pm 1 \times 10^{-3}$. The CYLTRAN benchmark was obtained using $10^{9}$ photon histories and forced photon collisions. The hybrid benchmark was obtained using $10^{9}$ photon histories in the Monte Carlo portion of the calculation with forced photon collisions and using 25 spatial cells, 100 energy groups, a 128 angle quadrature for the charged particles, and a 1024 angle quadrature for the photons in the $S_{N}$ portion of the calculation. The hybrid benchmark used 128 cross section moments for the electrons, positrons, and photons.

In Fig. 15, we show the relative error in $\hat{D}(z)$ for the Monte Carlo and hybrid methods 
for $10 \mathrm{MeV}$ photons on $0.1 \mathrm{~cm}$ of copper. We show the relative error in $\hat{r}(z)$ as a function of computation time in Fig. 16. The Monte Carlo portion of the hybrid calculations was performed with $10^{6}$ photon histories for calculations corresponding to 8,16 , and 24 quadrature in the $S_{N}$ portion of the calculation and $10^{7}$ photon histories for the remaining calculations for the 25 and 100 energy group calculations in which the $\mathrm{S}_{\mathrm{N}}$ quadrature was varied. In the two series of hybrid calculations using either a constant 32 electron quadrature or a constant 512 photon quadrature, $10^{6}$ incident Monte Carlo photons were followed, 25 logarithmic energy groups were used, and the photon or electron quadratures were varied, respectively. The $S_{N}$ portion of the hybrid calculations used 25 spatial cells. In all calculations, ONEBFP used as many cross section moments as could be accurately employed for the electrons and positrons and an equal number of cross section moments for the photons, even if a higher photon quadrature was used. The ONEBFP portion of hybrid calculations used four-moment MPSA except for the 25 energy group cases with 96 and 128 order quadrature, for which four-moment MPSA was unstable and $\mathrm{S}_{2}$ acceleration was used.

In Fig. 15, we again observe that the transversely integrated energy deposition is jointly dependent upon the quadrature order and the number of energy groups employed. In Fig. 16, we observe that an accurate calculation of the rms radius of the energy deposition requires a high-order photon quadrature. This is due to the fact that energy deposition at large radius results from bremsstrahlung and annihilation photons. Only those photons going in directions close to $\mu=0$ are unlikely to leak from the thin slab before undergoing an interaction. To accurately model the anisotropy of this secondary photon flux that contributes to the energy deposition at large radius, it is necessary to use a high-order angular quadrature set. However, calculation of the photon transport is inexpensive as compared to the charged particle transport, and we are not stongly penalized by resorting to very high-order quadratures.

Once more, we have found that by properly choosing the problem discretization, the radial moment calculations can be performed more efficiently using the hybrid method with 1-D calculations than the 3-D Monte Carlo method. To achieve a $5 \%$ relative error in the rms radius calculation, the hybrid method requires approximately $80 \mathrm{~s}$ while the Monte Carlo method requires approximately $1,500 \mathrm{~s}$. 


\section{V.D. Phosphor Screen}

Finally, we apply the radial moments calculations to an application of interest in the fields of industial radiography and medical imaging . ${ }^{30-34}$ In these two fields, high-energy photons passing through a "target" are used to produce an image. The target may be a weld in industrial radiography or a patient in medical imaging, but we will consider only a portion of the imaging problem. When bremsstrahlung photon beams are used with energies in the $\mathrm{MeV}$ to 10 's of $\mathrm{MeV}$ range, the photons cannot be recorded directly onto film. Instead, a "converter" must be employed. This converter may take the form of a phosphor screen that converts the high-energy photons into visible photons. ${ }^{34}$

In this section, we examine a typical phosphor screen. The incident photons interact with the screen and produce secondary electrons and positrons. These charged particles deposit energy in the phosphor and cause the screen to phosphoresce, yielding visible photons that can be recorded onto film after escaping from the screen.

The phosphor screen employed in the imaging process can be optimized for the application. A tradeoff occurs between the strength and the resolution of the image. A thicker screen results in more photon interactions and allows electrons to transfer more energy to the medium before escaping. This results in greater production of visible photons and can produce a stronger image. However, a thicker screen also results in spreading of the radiation, both in the energy deposited by the charged particles and the visible photons escaping from the screen. This degrades the resolution.

A feature used to enhance light production is a metal plate on the incident edge of the screen. ${ }^{32}$ A material with high density, such as copper or tungsten, induces more incident photon interactions and can produce a cascade of charged particles into the phosphor. As in the phosphor, a similar tradeoff can be evaluated in optimizing the thickness of the metal plate.

We have demonstrated that we can efficiently calculate the transverse energy deposition and the root-mean-squared radial spread of the energy deposition for this problem using the modified ONEBFP code. We can evaluate the mean energy deposition and mean loss of resolution resulting from the transport interactions of photons, electrons, and positrons by considering a unit normal pencil beam. In the quantum accounting formalism employed by 
Bissonnette et al., ${ }^{33}$ this corresponds to Stages 1 and 2.

To demonstrate the applicability of the radial moments method in ONEBFP to such a problem, we evaluate a screen with layers of $0.1 \mathrm{~cm}$ of copper and $0.4 \mathrm{~cm}$ of gadolinium oxysulfide $\left(\mathrm{Gd}_{2} \mathrm{O}_{2} \mathrm{~S}\right)$, a commonly used phosphor, with a reduced density of $3.67 \mathrm{~g} / \mathrm{cm}^{3}{ }^{32}$ We consider a monoenergetic $10 \mathrm{MeV}$ photon pencil beam normally incident on the screen at zero radius. The energy deposition as a function of depth is plotted in Fig. 17, and the rms radius of the energy deposition is plotted in Fig. 18.

The relative difference in the energy deposition distributions between the hybrid benchmark and the Monte Carlo benchmark is $6.31 \times 10^{-3} \pm 8 \times 10^{-5}$. The relative difference in the rms radius of the energy deposition is $3.24 \times 10^{-2} \pm 9 \times 10^{-4}$.

For high-energy beams, we have observed that the rms radii of the energy deposition calculated with ONEBFP or the hybrid method are consistently smaller than the CYLTRAN results by approximately $3 \%$. The cause of this is unclear. However, the rms radius in these problems is strongly dependent on contributions to the energy deposition at large radius from bremstrahlung and annihilation photons. Small differences in the production of these photons may be the cause of the discrepency.

The CYLTRAN benchmark was obtained using $10^{8}$ photon histories and forced photon collisions. The hybrid benchmark was obtained using $10^{9}$ photon histories in the Monte Carlo portion of the calculation with the same forced interactions as used in the CYLTRAN benchmark and using 25 spatial cells, 100 energy groups, 128 angle quadrature for the charged particles, 1024 angle quadrature for the photons, and 128 cross section moments for all particles in the $S_{N}$ portion of the calculation.

In Fig. 19, we show the relative error in $\hat{D}(z)$ for the Monte Carlo and hybrid methods in the metal plate and phosphor screen. We show the relative error in $\hat{r}(z)$ as a function of computation time in Fig. 20. The CYLTRAN efficiency data in these figures was generated using increasing numbers of incident photon histories.

In addition, for a series of CYLTRAN calculations using $10^{6}$ incident photons, the "SCALE-BREMS" feature was used to enhance the production of bremsstrahlung photons. We found that, while accurately modeling the secondary photons was crucial to the $S_{N}$ radial moment calculations, increasing the bremsstrahlung production in CYLTRAN is only 
slightly beneficial for calculating the rms radius of the energy deposition. It is possible that preferential production of secondary photons in directions close to $\mu=0$ with correspondingly smaller weights would have been an effective variance reduction method, but we do not examine that possibility here.

For the hybrid calculations, the Monte Carlo portion was performed with $10^{6}$ photon histories for the calculations corresponding to 8,16 , and 24 quadrature in the $\mathrm{S}_{\mathrm{N}}$ portion of the calculation and $10^{7}$ photon histories for the remaining calculations for 25 and 100 energy groups, in which the $S_{N}$ quadrature was varied. In the hybrid calculations using either 32 electron quadrature or 512 photon quadrature, $10^{6}$ incident Monte Carlo photons were followed, 25 logarithmic energy groups were used, and the photon or electron quadratures were varied, respectively. The ONEBFP portions of the hybrid calculations all used 25 spatial cells and used four-moment MPSA for all except the 25 energy group cases with 96 and 128 order quadrature, for which four-moment MPSA was unstable and $S_{2}$ acceleration was used.

From Fig. 20 we find that with two minutes of computation time we can acquire an accurate calculation of the energy deposition and the rms radius of the energy deposition in a phosphor screen. At such a low cost, a wide variety of thicknesses and types of phosphor screens can be analyzed quickly.

\section{CONCLUSIONS}

In this work we have developed an efficient 1-D method for calculating exact information about multidimensional radiation beams. We have derived 1-D radial moment transport equations and adapted the ONEBFP discrete ordinates code to solve the radial moments transport equations up to the second-order radial moment. To speed up the iterative convergence of the discrete ordinates calculations, we have implemented modified $P_{N}$ synthetic acceleration (MPSA) in ONEBFP. Acceleration of 4 flux moments with MPSA was found to produce approximately a factor of 2 speedup beyond $\mathrm{S}_{2}$ acceleration.

We have demonstrated the efficiency of the 1-D method for acquiring realistic calculations of the rms radius of the energy deposition resulting from electron and photon beams. We have analyzed the sensitivity of the accuracy of calculations to refinements in the spatial, 
angular, and energy discretization and compared the efficiency of the method to Monte Carlo calculations using the ITS CYLTRAN code. We found that a hybrid Monte Carlo/discrete ordinates method is effective for high-energy photon beams and that a high-order photon quadrature is necessary to calculate accurate radial information in thin slabs.

Finally, we discern a number of possible enhancements to this method. First, we have used the solutions of the "second" equations only as a source in the "third" equations [(16)(18)]. However, these solutions contain physically relevant information - the first radial moments of the net radial currents. These quantities cannot be directly converted to dose information, but useful data might be extracted from them at little extra cost. Second, the radial moments could be employed in a polynomial, exponential, or other analytical expression to present a more complete description of the radial distribution of the energy deposition. Third, the radial moments information could be incorporated into a figure of merit for the optimization of phosphor screens used in radiography. For such applications the "noise" (statistical variance in energy deposition due to the small number of incident photon interactions) is also of interest. Radial moment equations of the variance in energy deposition could be derived from the stochastic transport equation and implemented. ${ }^{35}$

\section{ACKNOWLEDGMENTS}

This work was supported by the Nuclear Engineering/Health Physics Fellowship Program of the U.S. Department of Energy and the Office of Nuclear Energy, Science and Technology. Sandia is a multiprogram laboratory operated by Sandia Corporation, a Lockheed Martin Company, for the United States Department of Energy under contract DE-AC04-94AL85000. 


\section{References}

[1] D.E. Bartine, R.G. Alsmiller, Jr., F.R. Mynatt, W.W. Engle, Jr., and J. Barish, "LowEnergy Electron Transport by the Method of Discrete Ordinates," Nucl. Sci. Eng. 48, $159 \cdot(1972)$.

[2] J.E. Morel, "On the Validity of the Extended Transport Cross-Section Correction for Low-Energy Electron Transport," Nucl. Sci. Eng. 71, 64 (1979).

[3] L.J. Lorence, Jr., J.E. Morel, and G.D. Valdez, "Results Guide to CEPXSONELD: A One-Dimensional Coupled Electron-Photon Discrete-Ordinates Code Package," SAND89-2211, Sandia National Laboratories (1990).

[4] K. Przybylski and J. Ligou, "Numerical Analysis of the Boltzmann Equation Including Fokker-Planck Terms," Nucl. Sci. Eng. 81, 92 (1982).

[5] J.E. Morel, "An Improved Fokker-Planck Angular Differencing Scheme," Nucl. Sci. Eng. 89, 131 (1985).

[6] K.M. Khattab and E.W. Larsen, "Synthetic Acceleration Methods for Linear Transport Problems with Highly Anisotropic Scattering," Nucl. Sci. Eng. 107, 217 (1991).

[7] J.E. Morel and T.A. Manteuffel, "An Angular Multigrid Acceleration Technique for $\mathrm{S}_{\mathrm{N}}$ Equations with Highly Forwarded-Peaked Scattering," Nucl. Sci. Eng. 107, 330 (1991).

[8] R.P. Datta, S.D. Altekar, A.K. Ray, and J.E. Morel, "Computational Model for Coupled Electron-Photon Transport in Two Dimensions," Phys. Rev. E 53, 6514 (1996).

[9] C.R. Drumm, "Multidimensional Electron-Photon Transport with Standard Discrete Ordinates Codes," Nucl. Sci. Eng. 127, 1 (1997).

[10] S.D. Pautz, "Discrete Ordinates Transport Methods for Problems with Highly ForwardPeaked Scattering," Ph.D. Dissertation, Texas A\&M University (1998).

[11] B. Rossi and K. Griesen, "Cosmic Ray Theory," Rev. Mod. Phys. 13, 265 (1941).

[12] L. Eyges, "Multiple Scattering with Energy Loss," Phys. Rev. 74, 1534 (1948). 
[13] D. Jette, "Electron Dose Calculations," Medical Radiology, Radiation Therapy Physics, edited by A.R. Smith, pp. 95-121, Springer-Verlag, Berlin (1995).

[14] G.C. Pomraning and A.K. Prinja, "Transverse Diffusion of a Collimated Particle Beam," J. Stat. Phys. 80, 625 (1995).

[15] C. Börgers and E.W. Larsen, "On the Accuracy of the Fokker-Planck and Fermi Pencil Beam Equations for Charged Particle Transport," Med. Phys. 23, 1749 (1996).

[16] G.C. Pomraning, "A Non-Gaussian Treatment of Radiation Pencil Beams," Nucl. Sci. Eng. 127, 182 (1997).

[17] G.C. Pomraning and A.K. Prinja, "The Pencil Beam Problem for Screened Rutherford Scattering," Nucl. Sci. Eng. 130, 1 (1.998).

[18] G.C. Pomraning, "Large Deflection Beam Transport with Screened Rutherford Scattering," Prog. Nucl. Energy 34, 377 (1999).

[19] E.W. Larsen, "The Linear Boltzmann Equation in Optically Thick Systems with Forward-Peaked Scattering," Prog. Nucl. Energy 34, 413 (1999).

[20] E.W. Larsen, "The Amplitude and Radius of Radiation Pencil Beams," Transp. Theory Stat. Phys. 26, 533 (1997).

[21] B.C. Franke, "1-D Radial Moment Calculations of 3-D Coupled Electron-Photon Beams," Ph.D. Dissertation, Department of Nuclear Engineering and Radiological Sciences, University of Michigan (1999).

[22] J. Jenness, "Multiprogrammatic and Institutional Computing: Tera Cluster," UCRLMI-126001, http://www.llnl.gov/sccd/lc/mic/tera_cluster.html, December 12, 1998, Lawrence Livermore National Laboratory.

[23] D.R. Marr, K.J. Adams, R.E. Prael, and R.E. Alcouffe, "Deterministic Proton Transport Solving a One Dimensional Fokker-Planck Equation," LA-UR-97-2687, Los Alamos National Laboratory (1997). 
[24] W.F. Walters, "The Relation Between Finite Element Methods and Nodal Methods in Transport Theory," Prog. Nucl. Energy 18, 21 (1986).

[25] L.J. Lorence, Jr., J.E. Morel, and E.W. Larsen, "An S2 Synthetic Acceleration Scheme for the One-Dimensional $\mathrm{S}_{\mathrm{N}}$ Equations with Linear Discontinuous Spatial Differencing," Nucl. Sci. Eng. 101, 341 (1989).

[26] L.J. Lorence, Jr., J.E. Morel, and G.D. Valdez, "User's Guide to CEPXS-ONELD: A One-Dimensional Coupled Electron-Photon Discrete-Ordinates Code Package," SAND89-1661, Sandia National Laboratories (1989).

[27] L.J. Lorence, Jr., J.E. Morel, and G.D. Valdez, "Physics Guide to CEPXS: A Multigroup Coupled Electron-Photon Cross-Section Generating Code," SAND89-1685, Sandia National Laboratories (1989).

[28] J.A. Halbleib, R.P. Kensek, T.A. Mehlhorn, G.D. Valdez, S.M Seltzer, and M.J. Berger, "ITS Version 3.0: The Integrated TIGER Series of Coupled Electron/Photon Monte Carlo Transport Codes," SAND91-1634, Sandia National Laboratories (1991).

[29] R.E. Alcouffe, "Diffusion Synthetic Acceleration Methods for the Diamond-Differenced Discrete-Ordinates Equations," Nucl. Sci. Eng. 64, 344 (1977).

[30] T. Radcliffe, G. Barnea, B. Wowk, R. Rajapakshe, and S. Shalev, "Monte Carlo Optimization of Metal/Phosphor Screens at Megavoltage Energies," Med. Phys. 20, 1161 (1993).

[31] B. Wowk, T Radcliffe, K.W. Leszczynski, S. Shalev, and R. Rajapakshe, "Optimization of Metal/Phosphor Screens for On-Line Portal Imaging," Med. Phys. 21, 227 (1994).

[32] D.A. Jaffray, J.J. Battista, A. Fenster, and P. Munro, "Monte Carlo Studies of X-Ray Energy Absorption and Quantum Noise in Megavoltage Transmission Radiography," Med. Phys. 22, 1077 (1995).

[33] J.-P. Bissonnette, I.A. Cunningham, and P. Munro, "Optimal Phosphor Thickness for Portal Imaging," Med. Phys. 24, 803 (1997). 
[34] J.H. Siewerdsen, L.E. Antonuk, Y. El Mohri, J. Yorkston, W. Huang, J.M. Boudry, and I.A. Cunningham, "Empirical and Theoretical Investigation of the Noise Performance of Indirect Detection, Active Matrix Flat-Panel Imagers (AMFPIs) for Diagnostic Radiology," Med. Phys. 24, 71 (1997).

[35] C.W. Gardiner, Handbook of Stochastic Methods for Physics, Chemistry, and the Natural Sciences, Springer-Verlag, New York, 2nd ed. (1985). 


\section{LIST OF FIGURES}

Figure 1: $\hat{D}$ versus $z$ for a $10 \mathrm{MeV}$ electron pencil beam normally incident on a $0.5 \mathrm{~cm}$ copper slab.

Figure 2: $\hat{r}$ versus $z$ for a $10 \mathrm{MeV}$ electron pencil beam normally incident on a $0.5 \mathrm{~cm}$ copper slab.

Figure 3: Relative error in $\hat{D}(z)$ versus computation time for a $10 \mathrm{MeV}$ electron pencil beam normally incident on a $0.5 \mathrm{~cm}$ copper slab.

Figure 4: Relative error in $\hat{r}(z)$ versus computation time for a $10 \mathrm{MeV}$ electron pencil beam normally incident on a $0.5 \mathrm{~cm}$ copper slab.

Figure 5: Maximum absolute relative change in the scalar flux as a function of computation time for one energy group of an $S_{32}$ calculation with a mildly anisotropic flux distribution.

Figure 6: Maximum absolute relative change in the scalar flux as a function of computation time for one energy group of an $S_{32}$ calculation with a highly anisotropic flux distribution.

Figure 7: Relative error in $\hat{D}(z)$ versus computation time for a $10 \mathrm{MeV}$ electron pencil beam normally incident on a $0.5 \mathrm{~cm}$ copper slab for various levels of acceleration.

Figure 8: Relative error in $\hat{r}(z)$ versus computation time for a $10 \mathrm{MeV}$ electron pencil beam normally incident on a $0.5 \mathrm{~cm}$ copper slab for various levels of acceleration.

Figure 9: $\hat{D}$ versus $z$ for a $50 \mathrm{MeV}$ photon pencil beam normally incident.on a $0.5 \mathrm{~cm}$ copper slab.

Figure 10: $\hat{r}$ versus $z$ for a $50 \mathrm{MeV}$ photon pencil beam normally incident on a $0.5 \mathrm{~cm}$ copper slab. 
Figure 11: Relative error in $\hat{D}(z)$ versus computation time for a $50 \mathrm{MeV}$ photon pencil beam normally incident on a $0.5 \mathrm{~cm}$ copper slab.

Figure 12: Relative error in $\hat{r}(z)$ versus computation time for a $50 \mathrm{MeV}$ photon pencil beam normally incident on a $0.5 \mathrm{~cm}$ copper slab.

Figure 13: $\hat{D}$ versus $z$ for a $10 \mathrm{MeV}$ photon pencil beam normally incident on a $0.1 \mathrm{~cm}$ copper slab.

Figure 14: $\hat{r}$ versus $z$ for a $10 \mathrm{MeV}$ photon pencil beam normally incident on a $0.1 \mathrm{~cm}$ copper slab.

Figure 15: Relative error in $\hat{D}(z)$ versus computation time for a $10 \mathrm{MeV}$ photon pencil beam normally incident on a $0.1 \mathrm{~cm}$ copper slab.

Figure 16: Relative error in $\hat{r}(z)$ versus computation time for a $10 \mathrm{MeV}$ photon pencil beam normally incident on a $0.1 \mathrm{~cm}$ copper slab.

Figure 17: $\hat{D}$ versus $z$ for a $10 \mathrm{MeV}$ photon pencil beam normally incident on a slab of 0.1 cm copper and $0.4 \mathrm{~cm}$ phosphor.

Figure 18: $\hat{r}$ versus $z$ for a $10 \mathrm{MeV}$ photon pencil beam normally incident on a slab of 0.1 $\mathrm{cm}$ copper and $0.4 \mathrm{~cm}$ phosphor.

Figure 19: Relative error in $\hat{D}(z)$ versus computation time for a $10 \mathrm{MeV}$ photon pencil beam normally incident on a slab of $0.1 \mathrm{~cm}$ copper and $0.4 \mathrm{~cm}$ phosphor.

Figure 20: Relative error in $\hat{r}(z)$ versus computation time for a $10 \mathrm{MeV}$ photon pencil beam normally incident on a slab of $0.1 \mathrm{~cm}$ copper and $0.4 \mathrm{~cm}$ phosphor. 


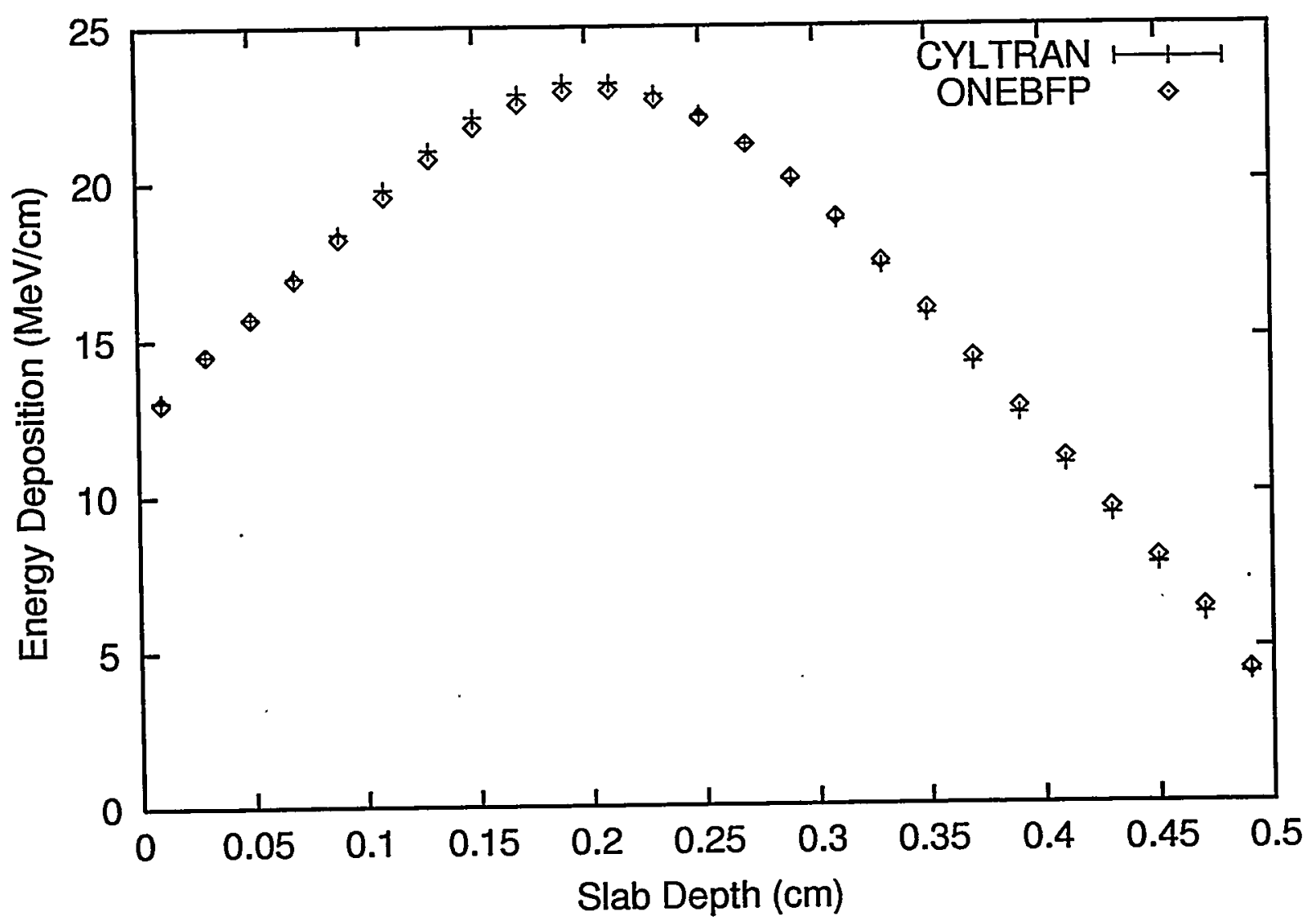

Figure 1: $\hat{D}$ versus $z$ for a $10 \mathrm{MeV}$ electron pencil beam normally incident on a $0.5 \mathrm{~cm}$ copper slab. 


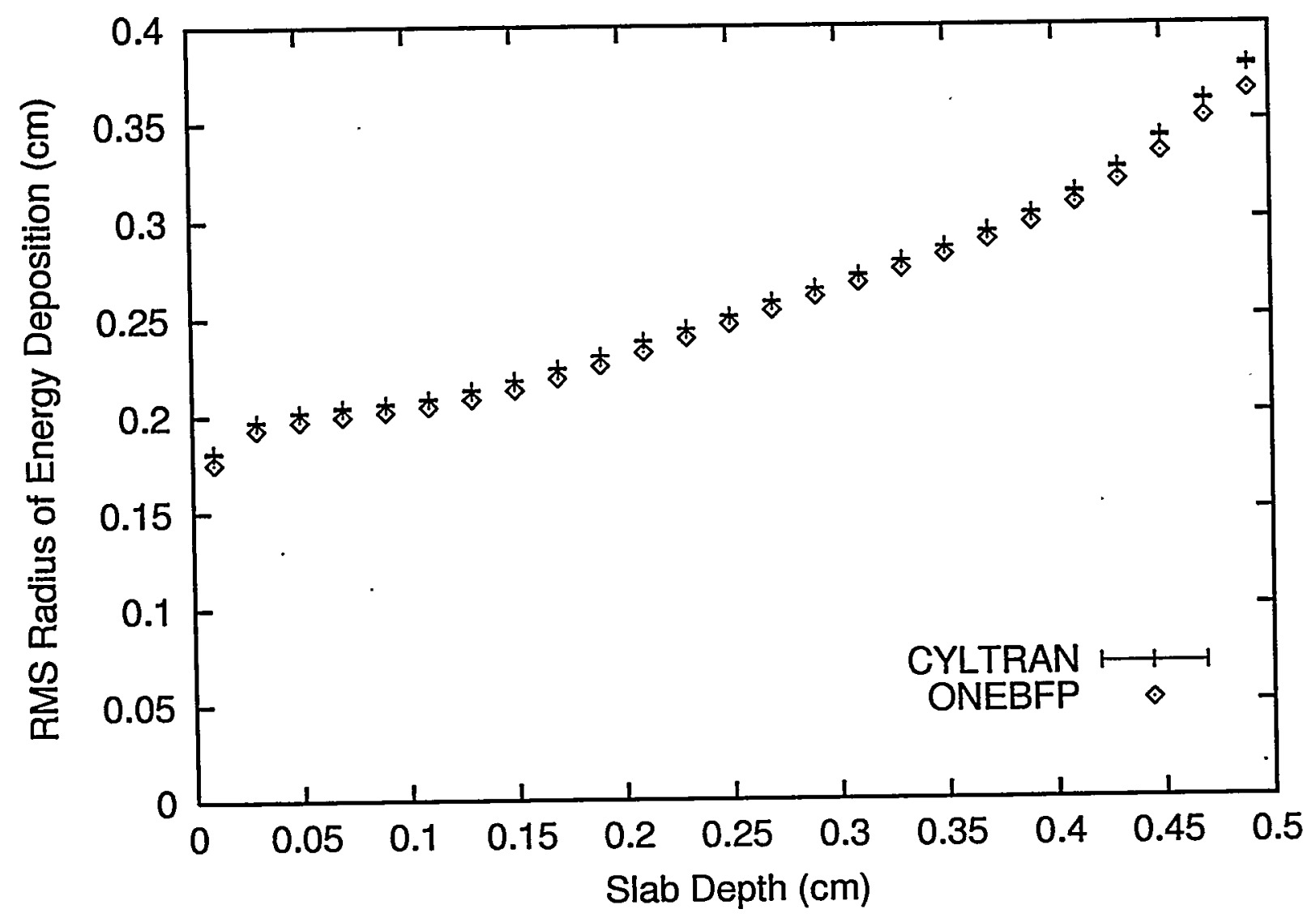

Figure 2: $\hat{r}$ versus $z$ for a $10 \mathrm{MeV}$ electron pencil beam normally incident on a $0.5 \mathrm{~cm}$ copper slab. 


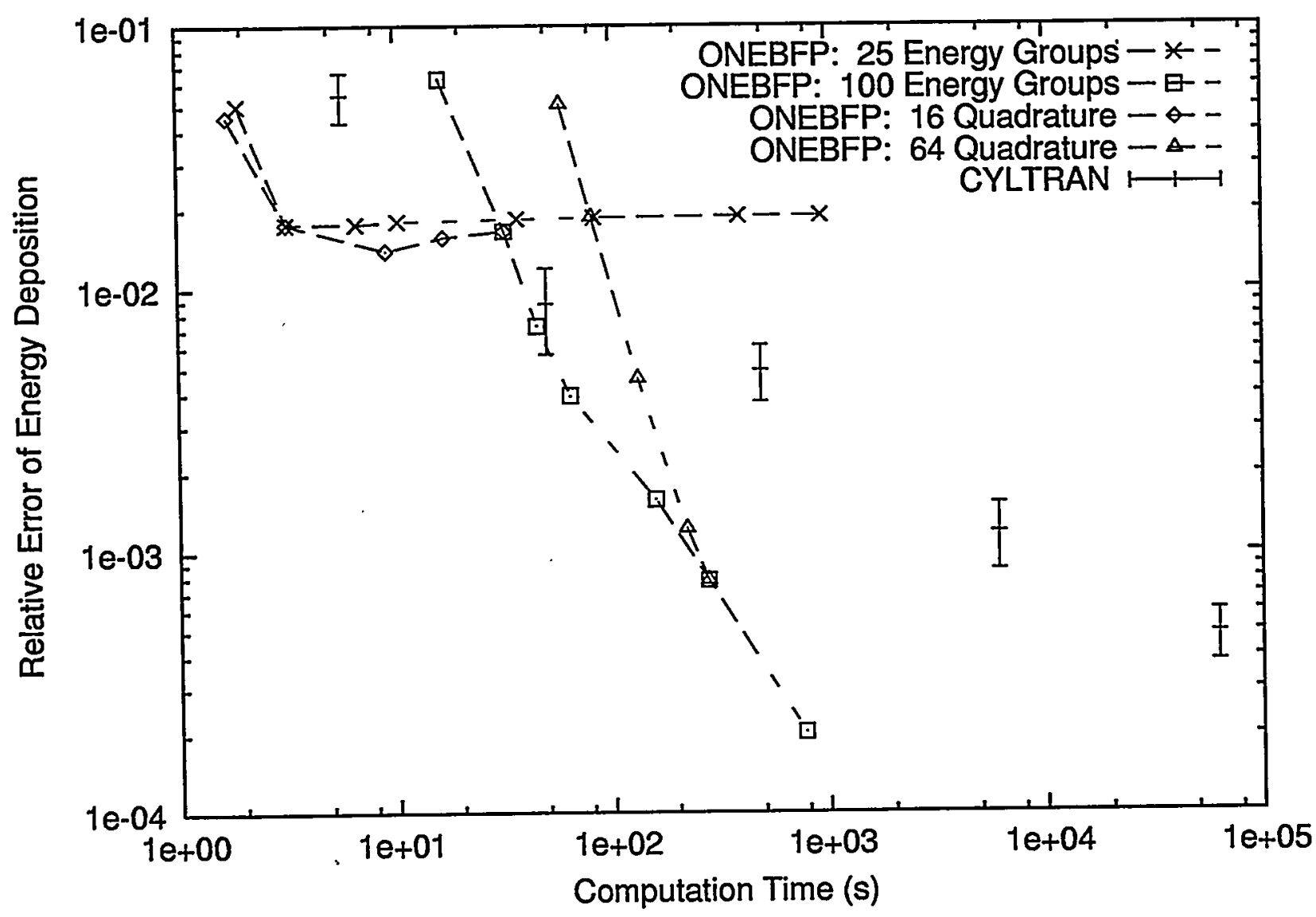

Figure 3: Relative error in $\hat{D}(z)$ versus computation time for a $10 \mathrm{MeV}$ electron pencil beam normally incident on a $0.5 \mathrm{~cm}$ copper slab. 


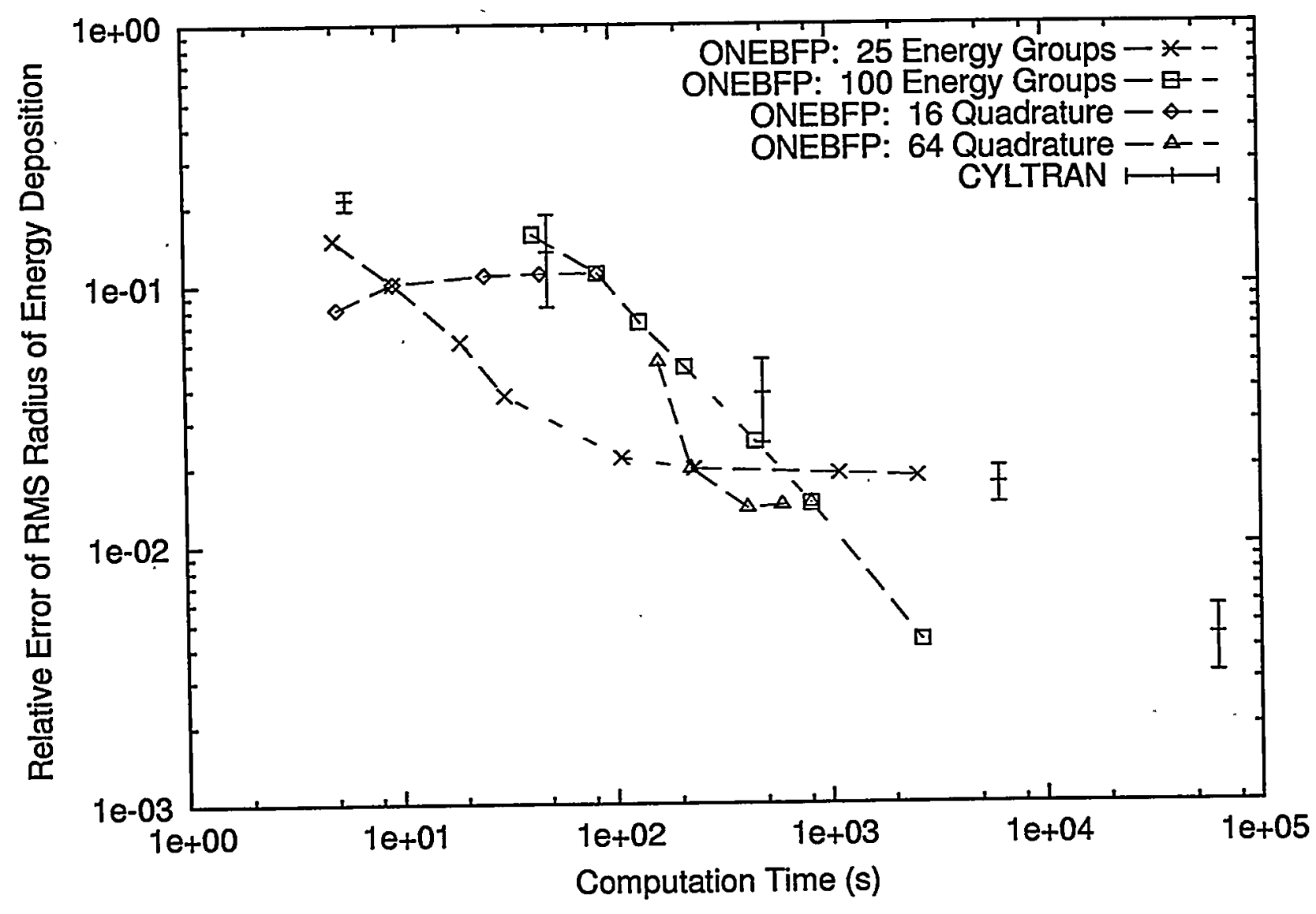

Figure 4: Relative error in $\hat{r}(z)$ versus computation time for a $10 \mathrm{MeV}$ electron pencil beam normally incident on a $0.5 \mathrm{~cm}$ copper slab. 


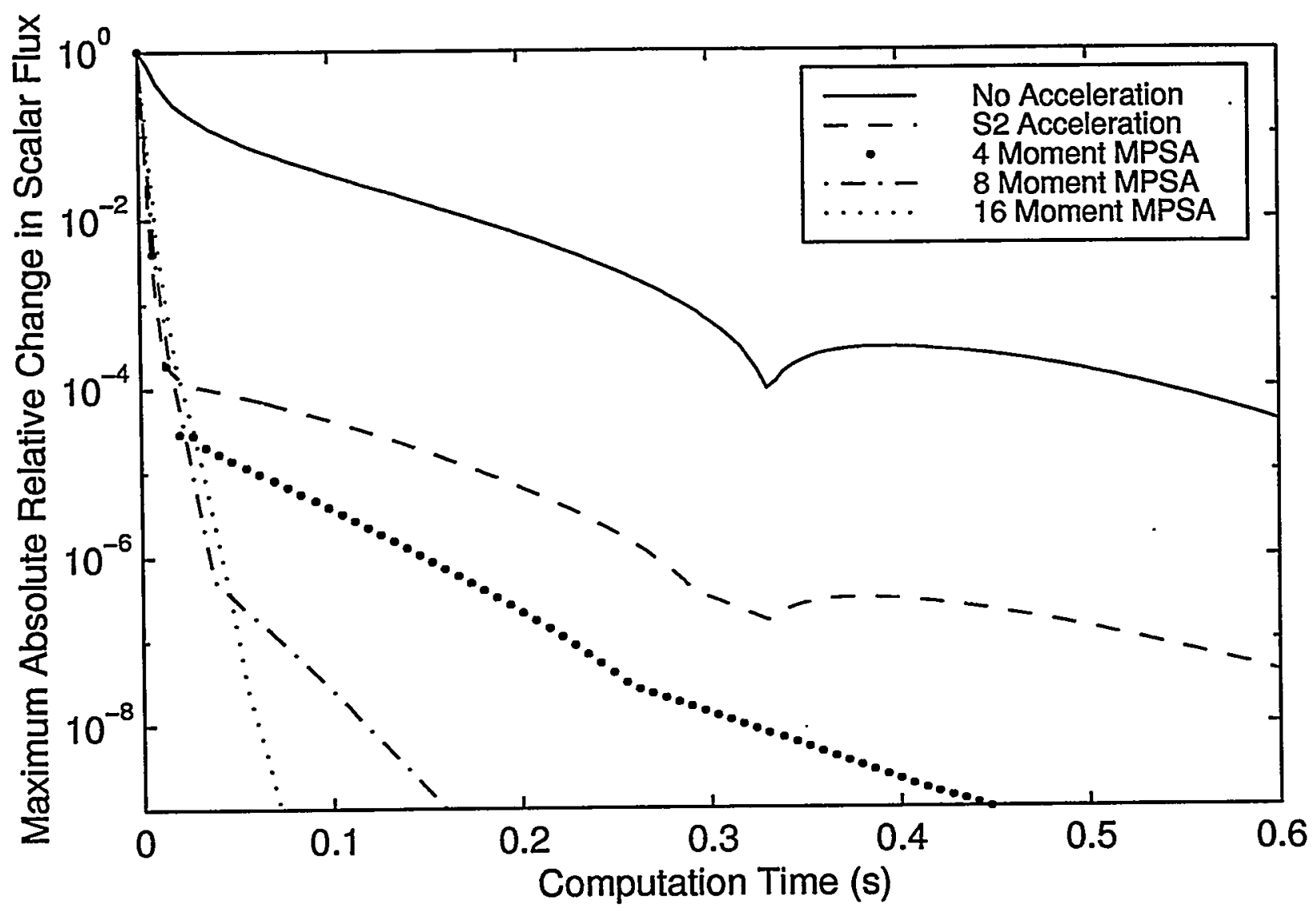

Figure 5: Maximum absolute relative change in the scalar flux as a function of computation time for one energy group of an $\mathrm{S}_{32}$ calculation with a mildly anisotropic flux distribution. 


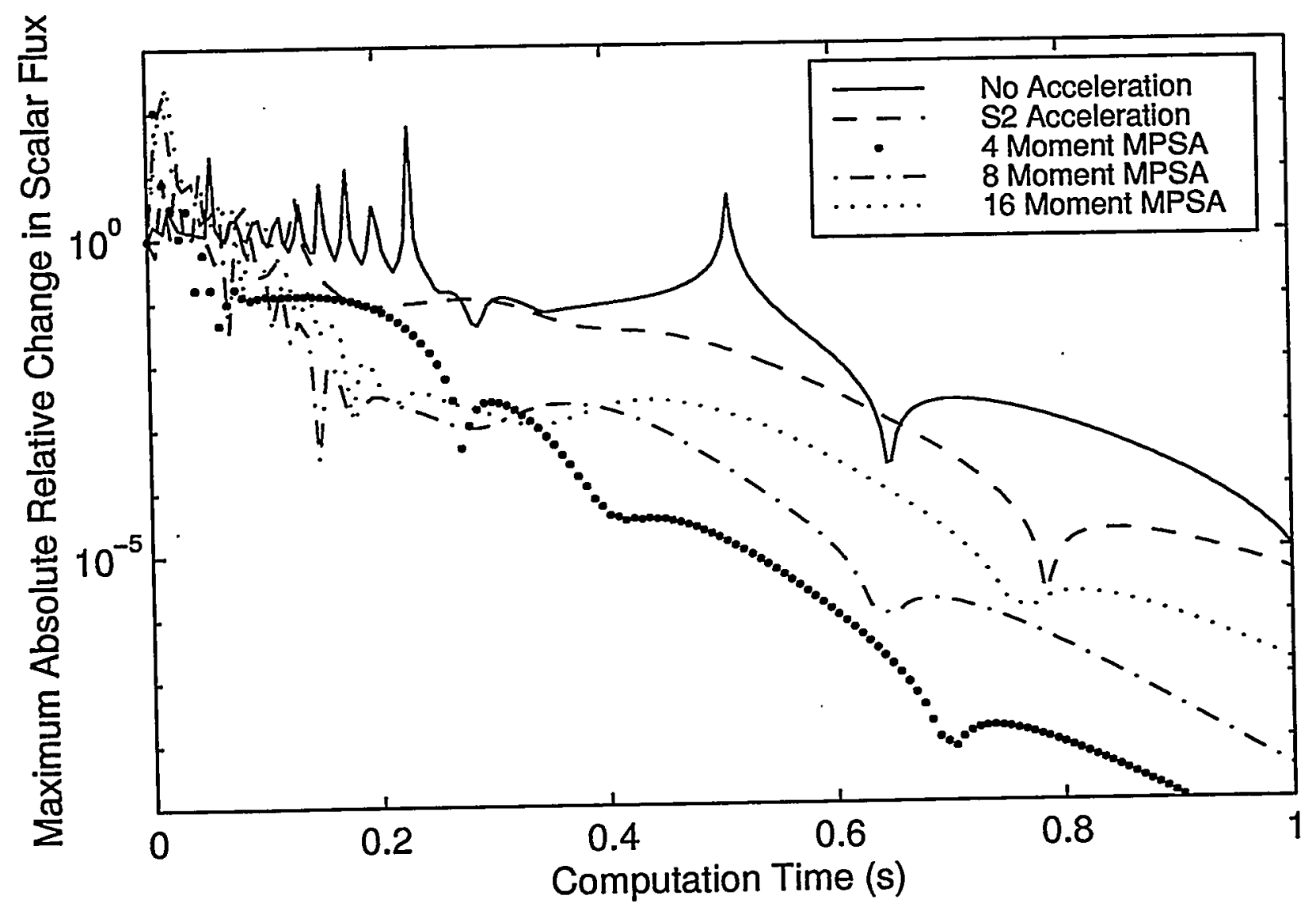

Figure 6: Maximum absolute relative change in the scalar flux as a function of computation time for one energy group of an $\mathrm{S}_{32}$ calculation with a highly anisotropic flux distribution. 


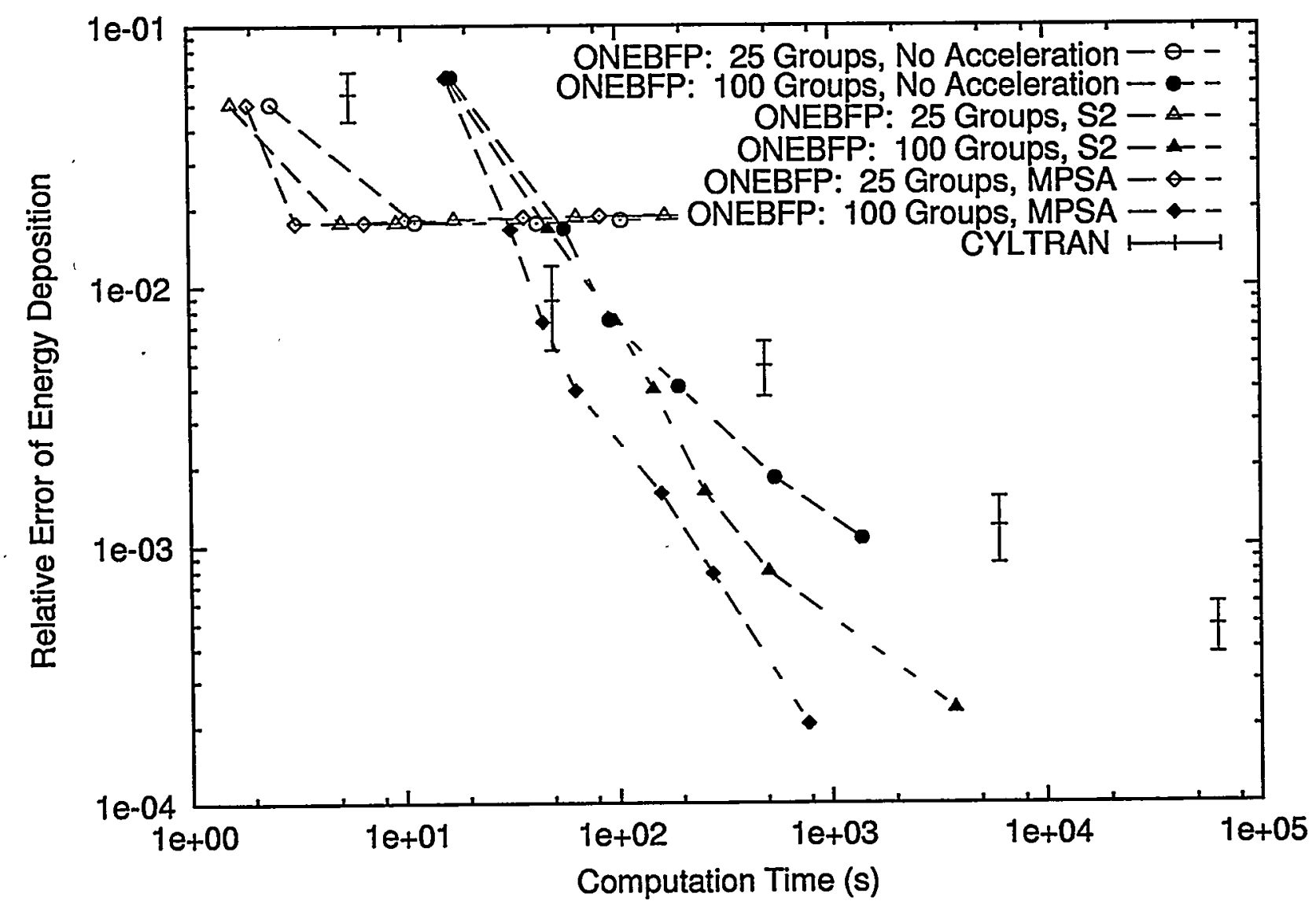

Figure 7: Relative error in $\hat{D}(z)$ versus computation time for a $10 \mathrm{MeV}$ electron pencil beam normally incident on a $0.5 \mathrm{~cm}$ copper slab for various levels of acceleration. 


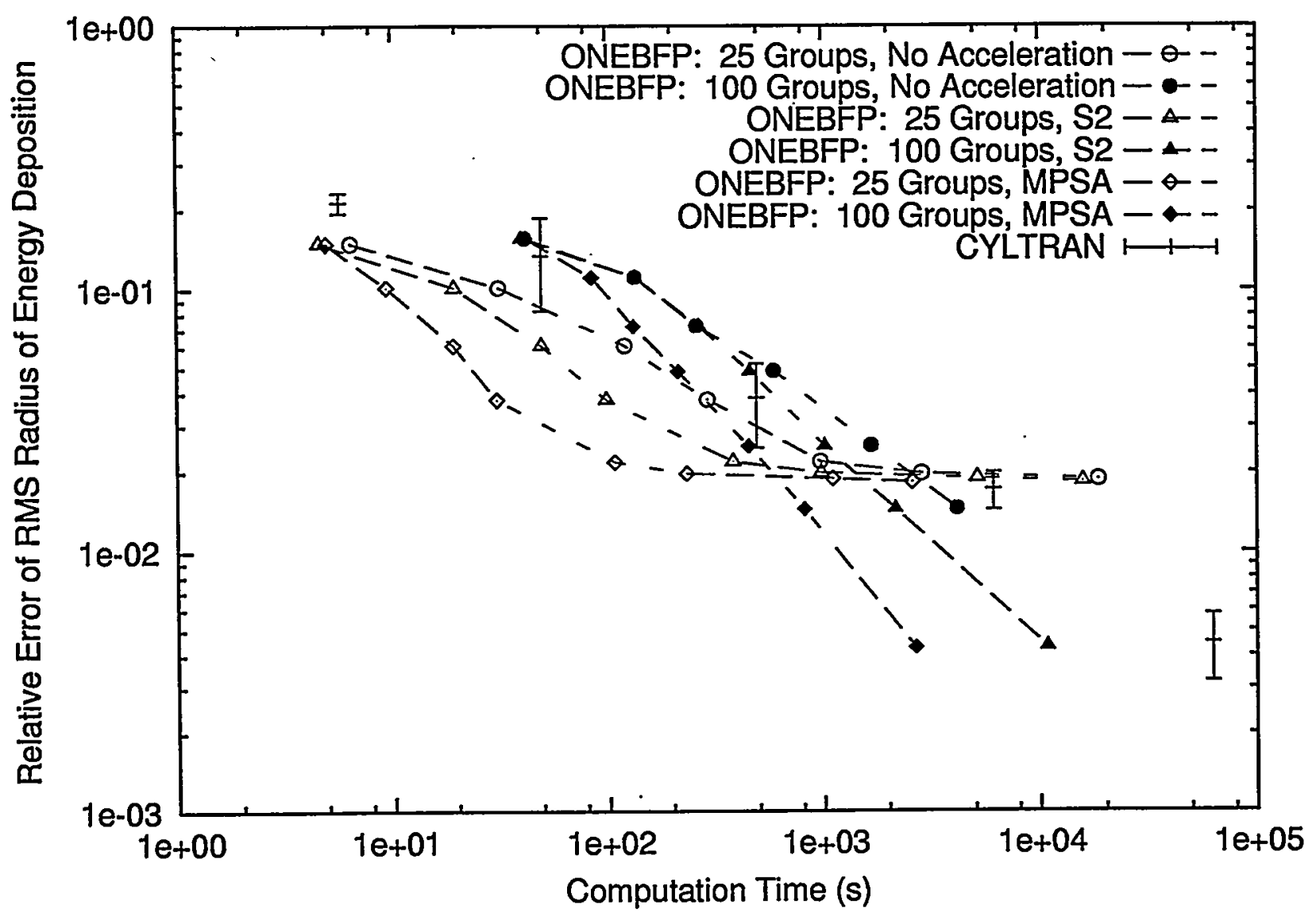

Figure 8: Relative error in $\hat{r}(z)$ versus computation time for a $10 \mathrm{MeV}$ electron pencil beam normally incident on a $0.5 \mathrm{~cm}$ copper slab for various levels of acceleration. 


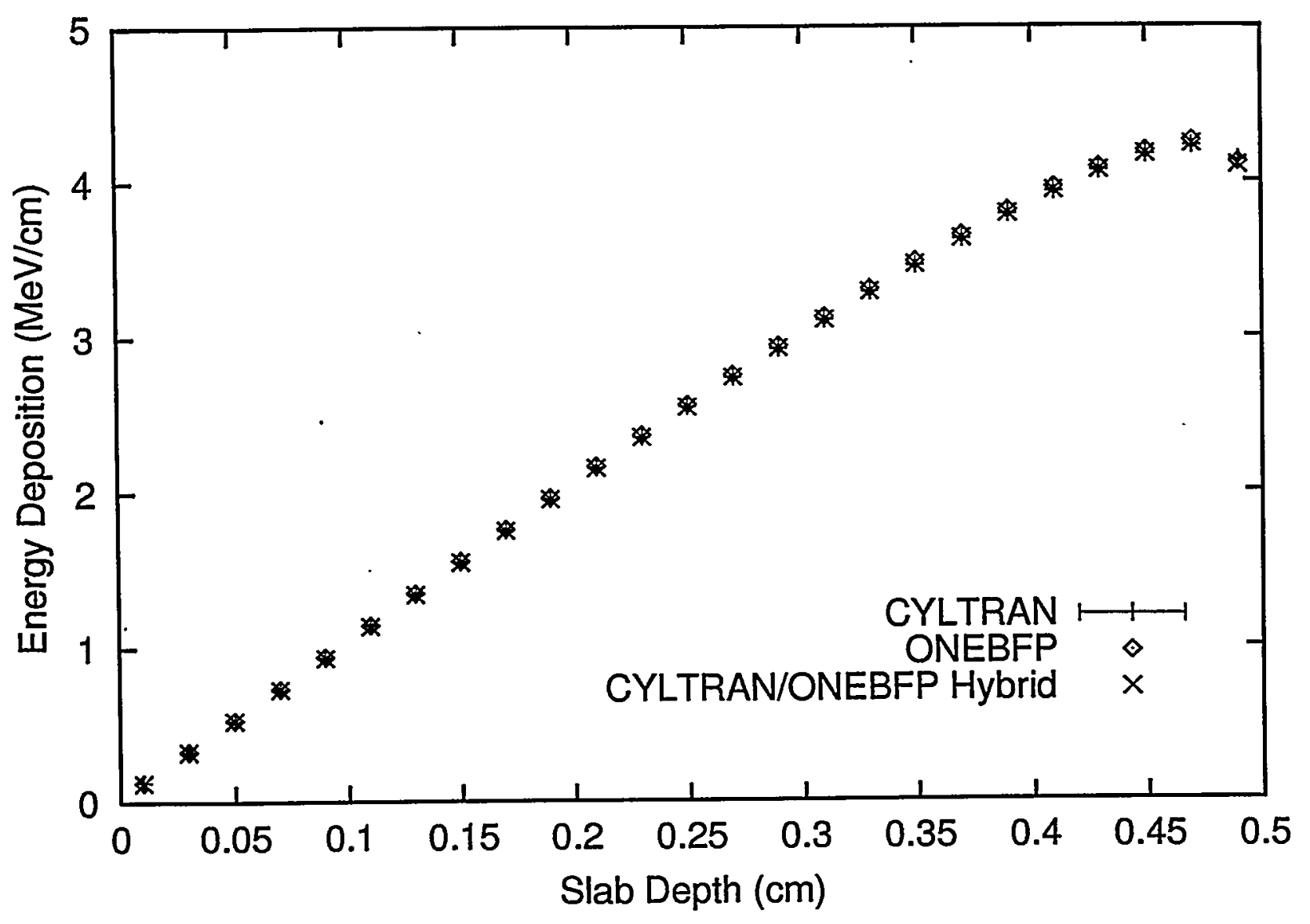

Figure 9: $\hat{D}$ versus $z$ for a $50 \mathrm{MeV}$ photon pencil beam normally incident on a $0.5 \mathrm{~cm}$ copper slab. 


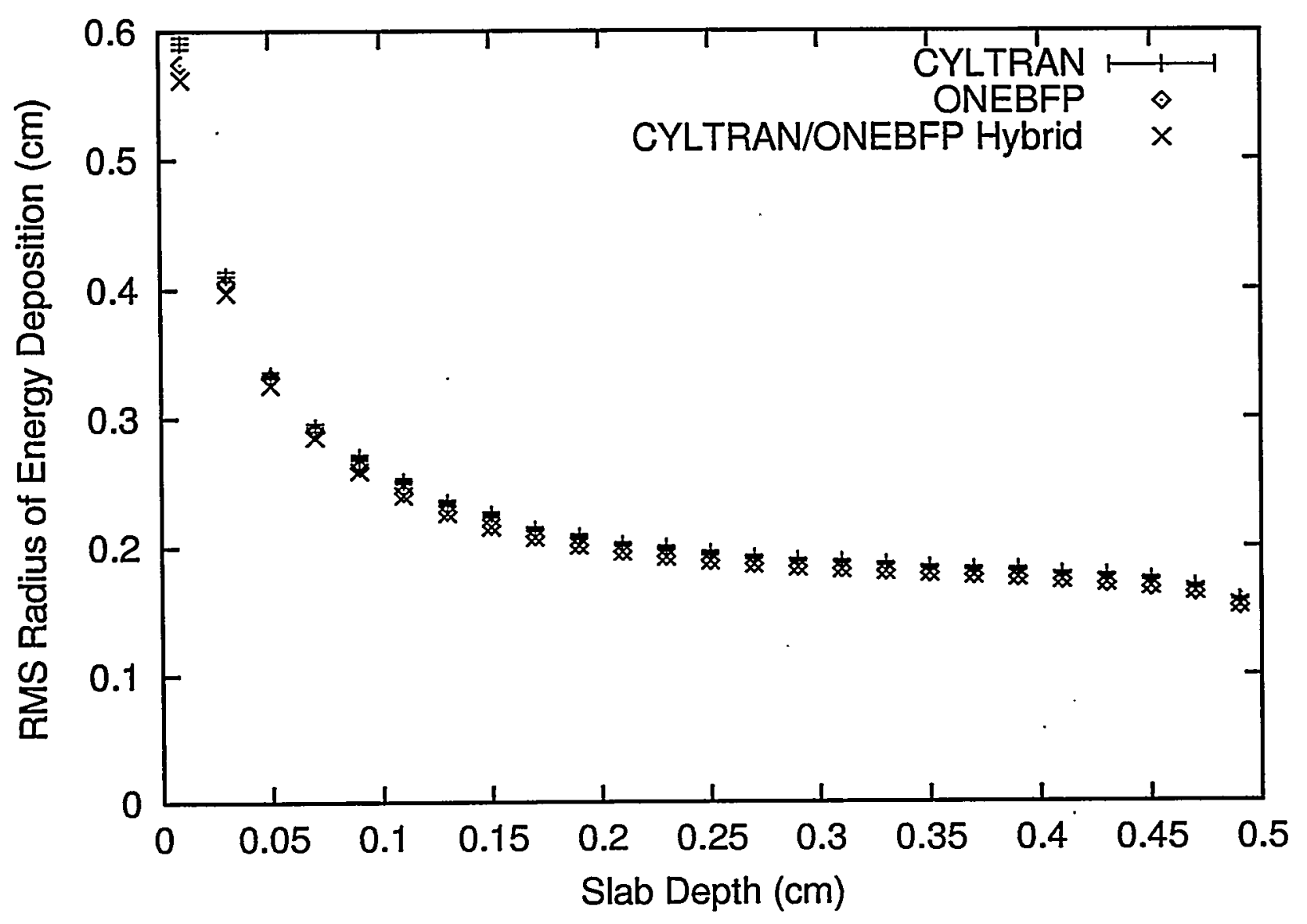

Figure 10: $\hat{r}$ versus $z$ for a $50 \mathrm{MeV}$ photon pencil beam normally incident on a $0.5 \mathrm{~cm}$ copper slab. 


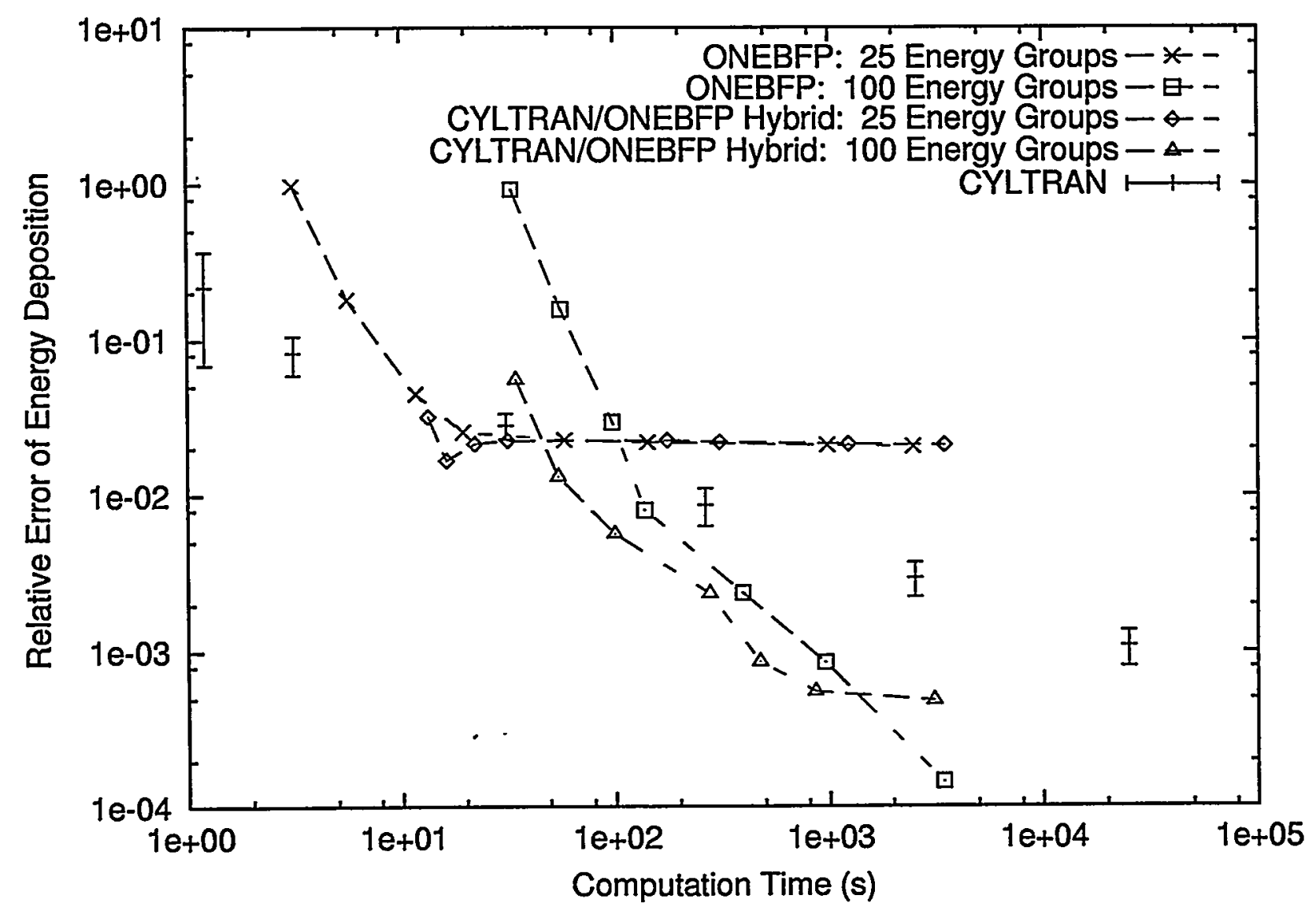

Figure 11: Relative error in $\hat{D}(z)$ versus computation time for a $50 \mathrm{MeV}$ photon pencil beam normally incident on a $0.5 \mathrm{~cm}$ copper slab. 


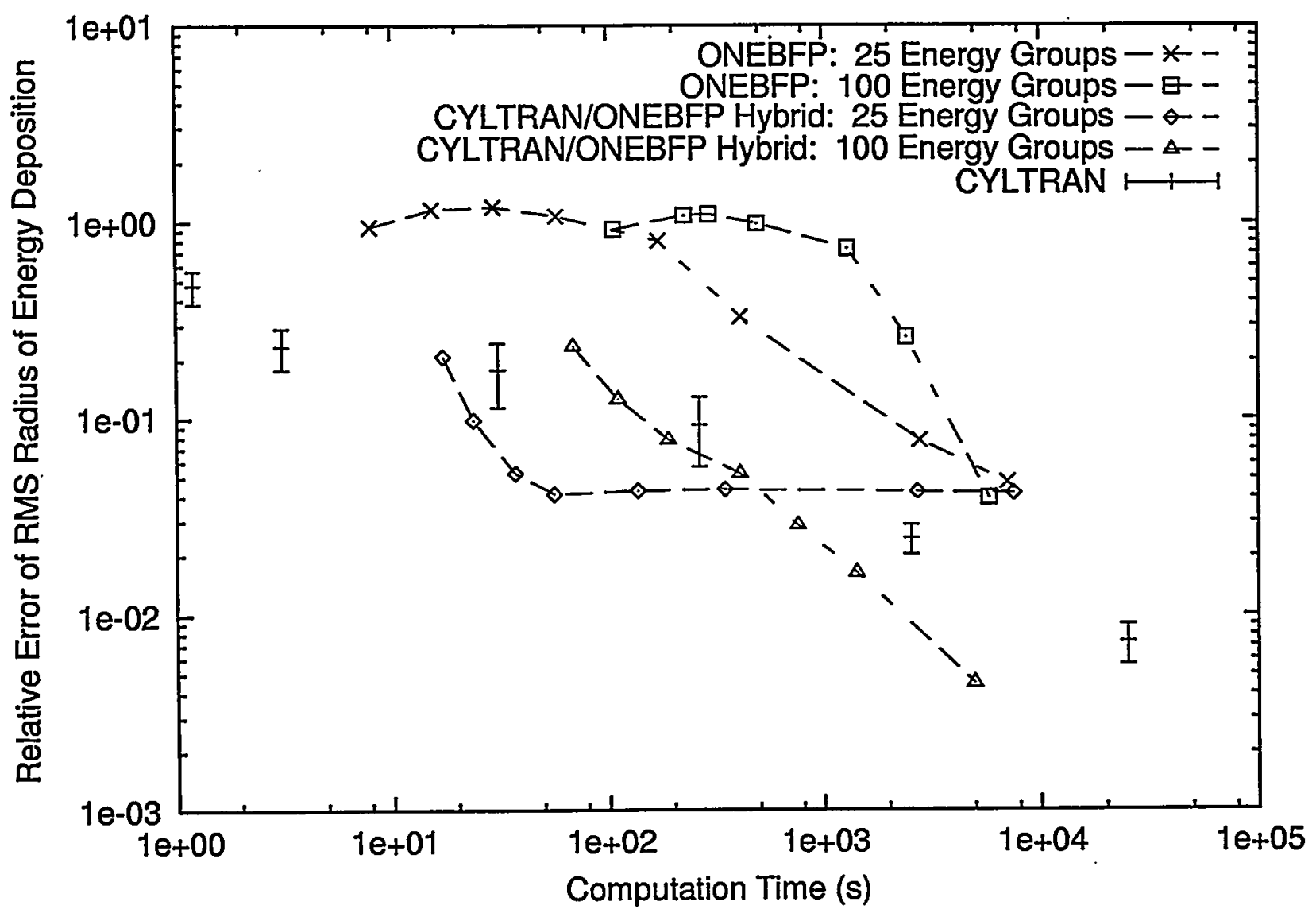

Figure 12: Relative error in $\hat{r}(z)$ versus computation time for a $50 \mathrm{MeV}$ photon pencil beam normally incident on a $0.5 \mathrm{~cm}$ copper slab. 


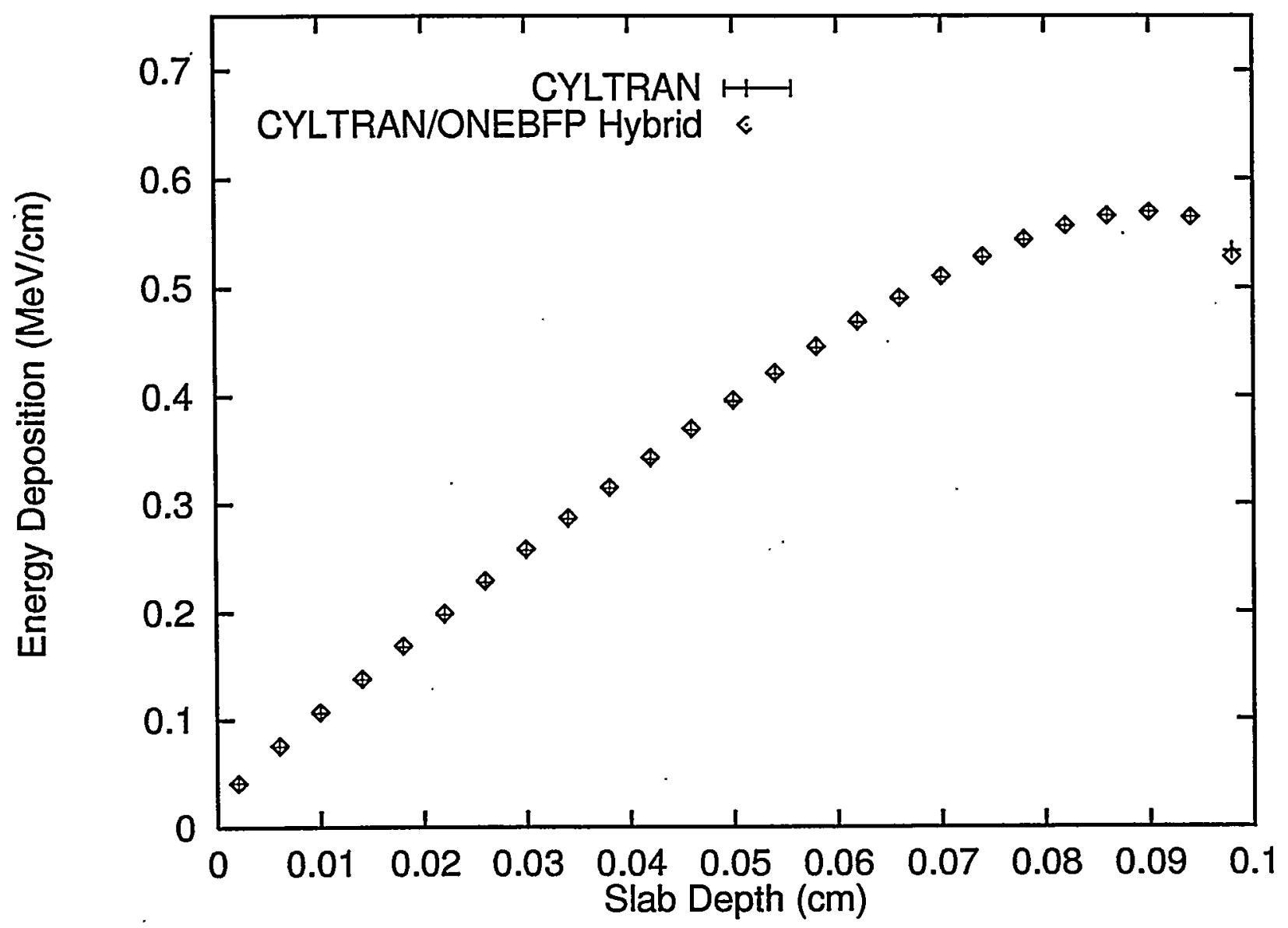

Figure 13: $\hat{D}$ versus $z$ for a $10 \mathrm{MeV}$ photon pencil beam normally incident on a $0.1 \mathrm{~cm}$ copper slab. 


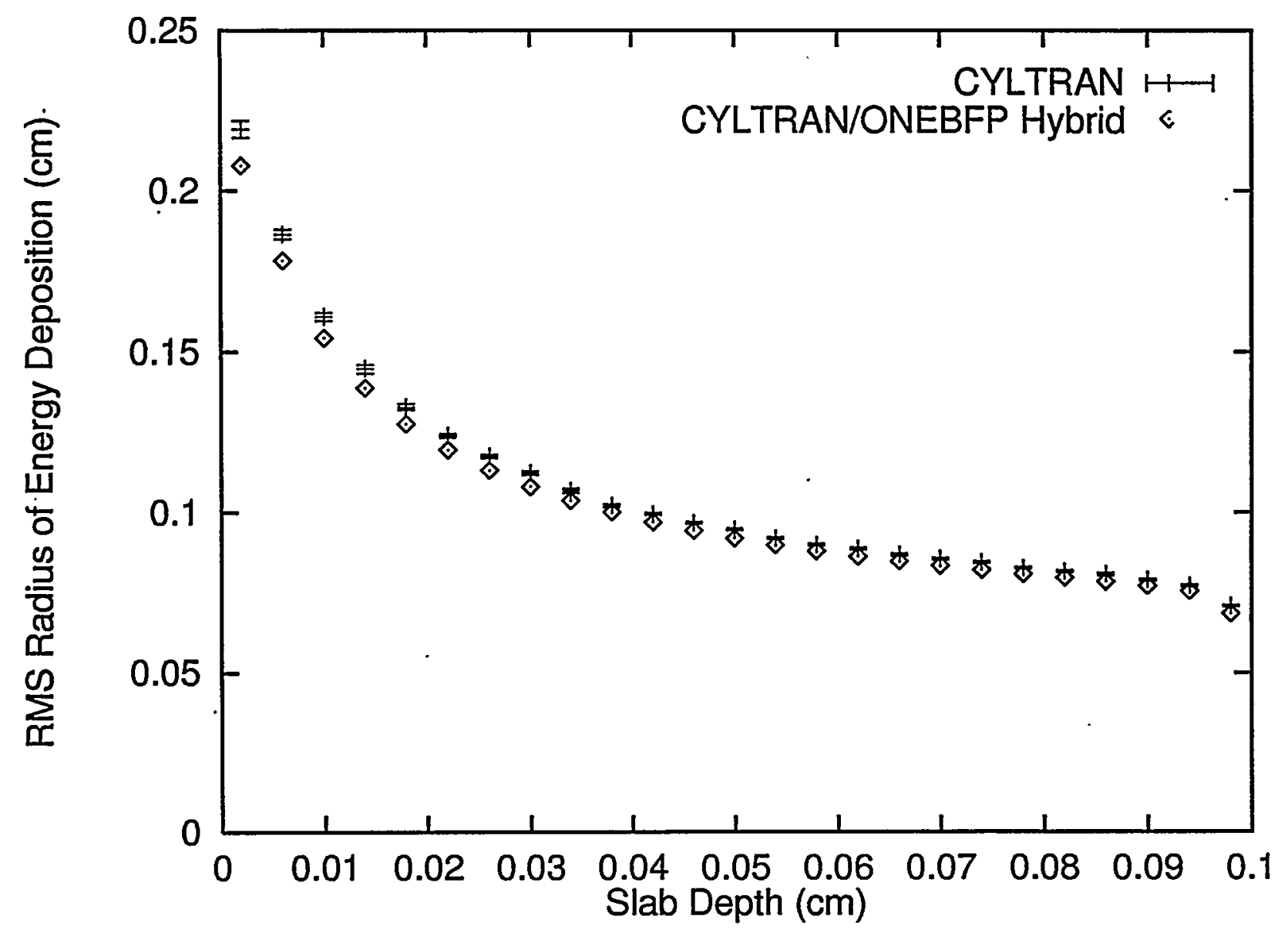

Figure 14: $\hat{r}$ versus $z$ for a $10 \mathrm{MeV}$ photon pencil beam normally incident on a $0.1 \mathrm{~cm}$ copper slab. 


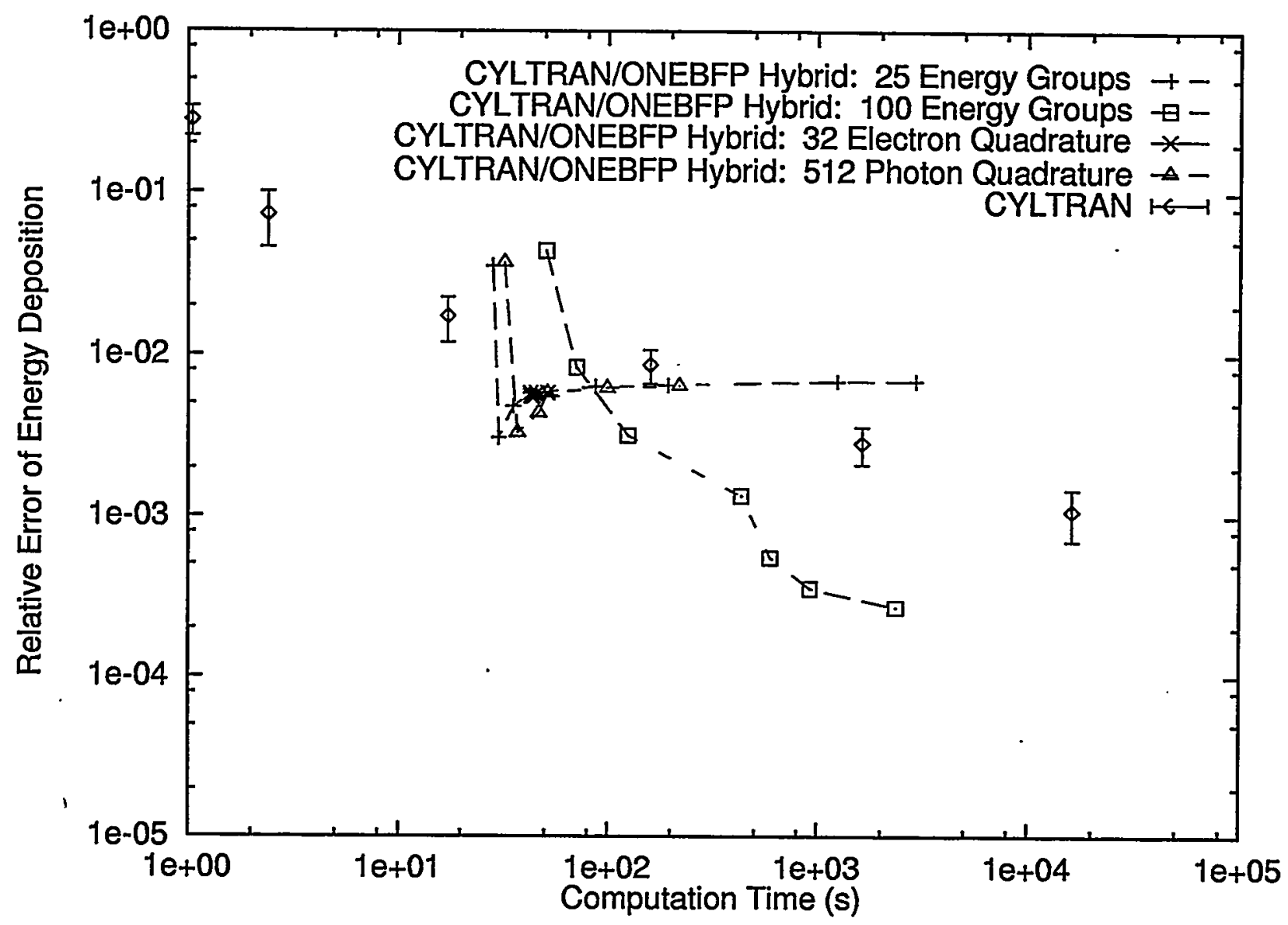

Figure 15: Relative error in $\hat{D}(z)$ versus computation time for a $10 \mathrm{MeV}$ photon pencil beam normally incident on a $0.1 \mathrm{~cm}$ copper slab. 


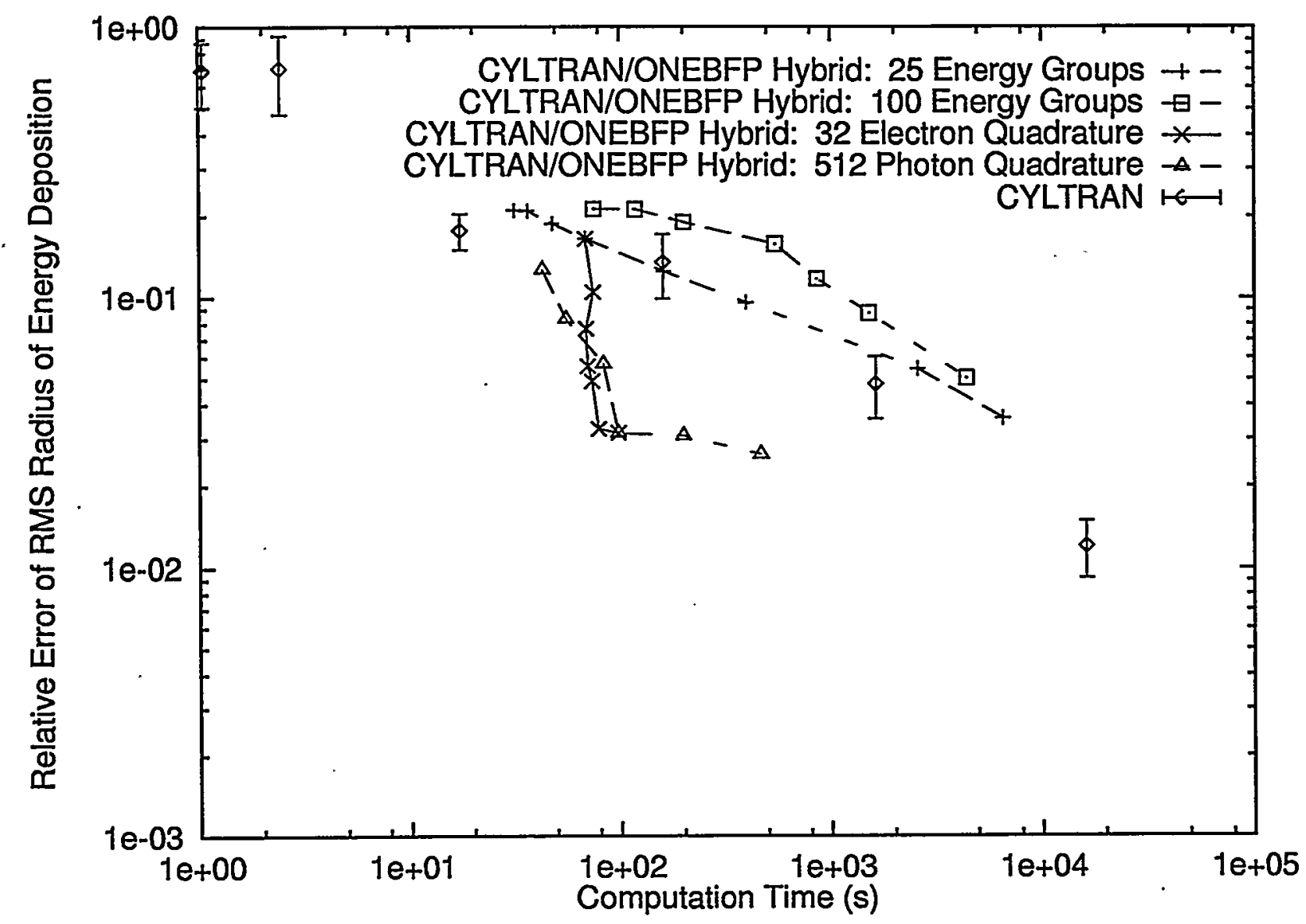

Figure 16: Relative error in $\hat{r}(z)$ versus computation time for a $10 \mathrm{MeV}$ photon pencil beam normally incident on a $0.1 \mathrm{~cm}$ copper slab. 


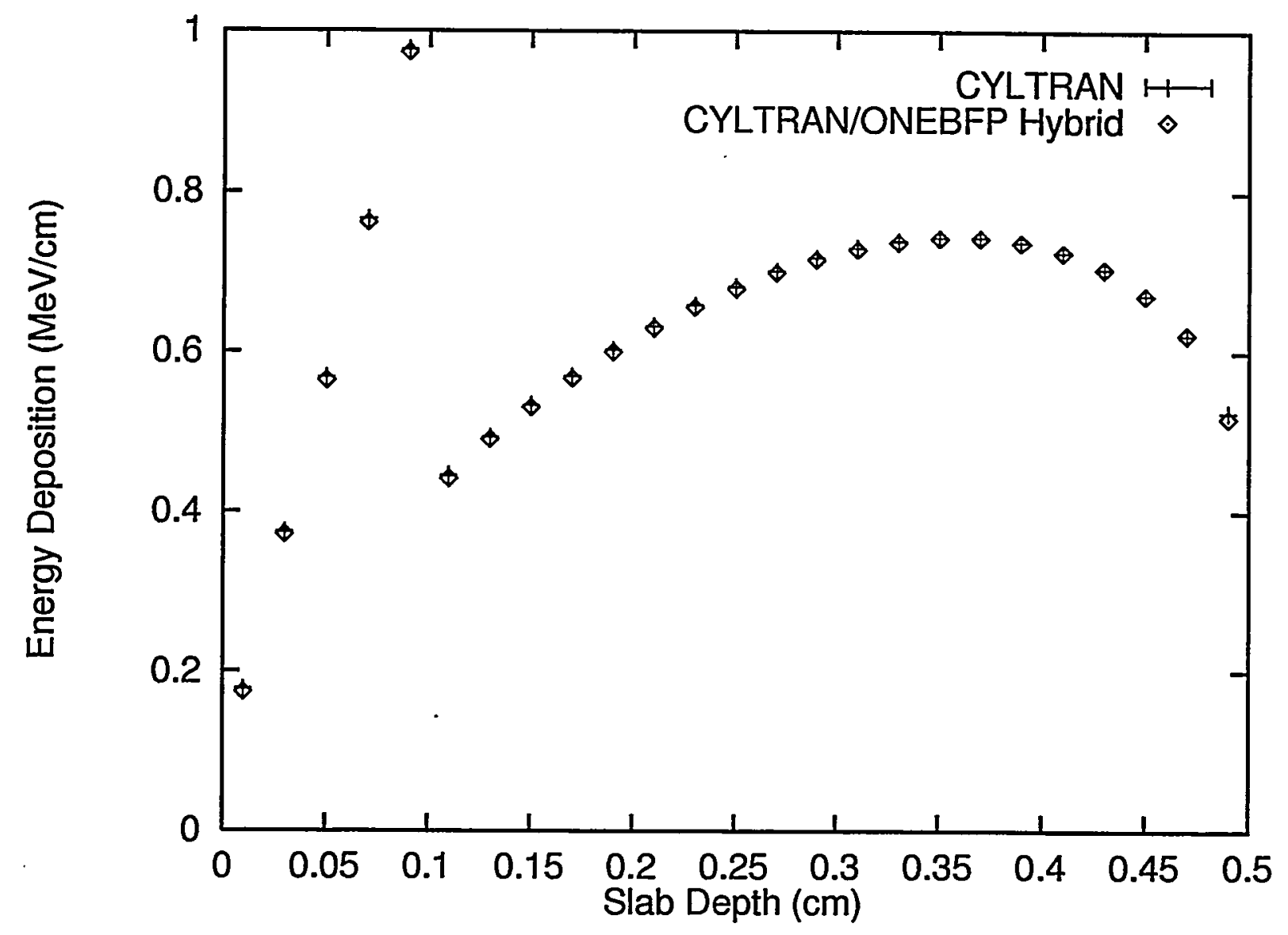

Figure 17: $\hat{D}$ versus $z$ for a $10 \mathrm{MeV}$ photon pencil beam normally incident on a slab of 0.1 cm copper and $0.4 \mathrm{~cm}$ phosphor. 


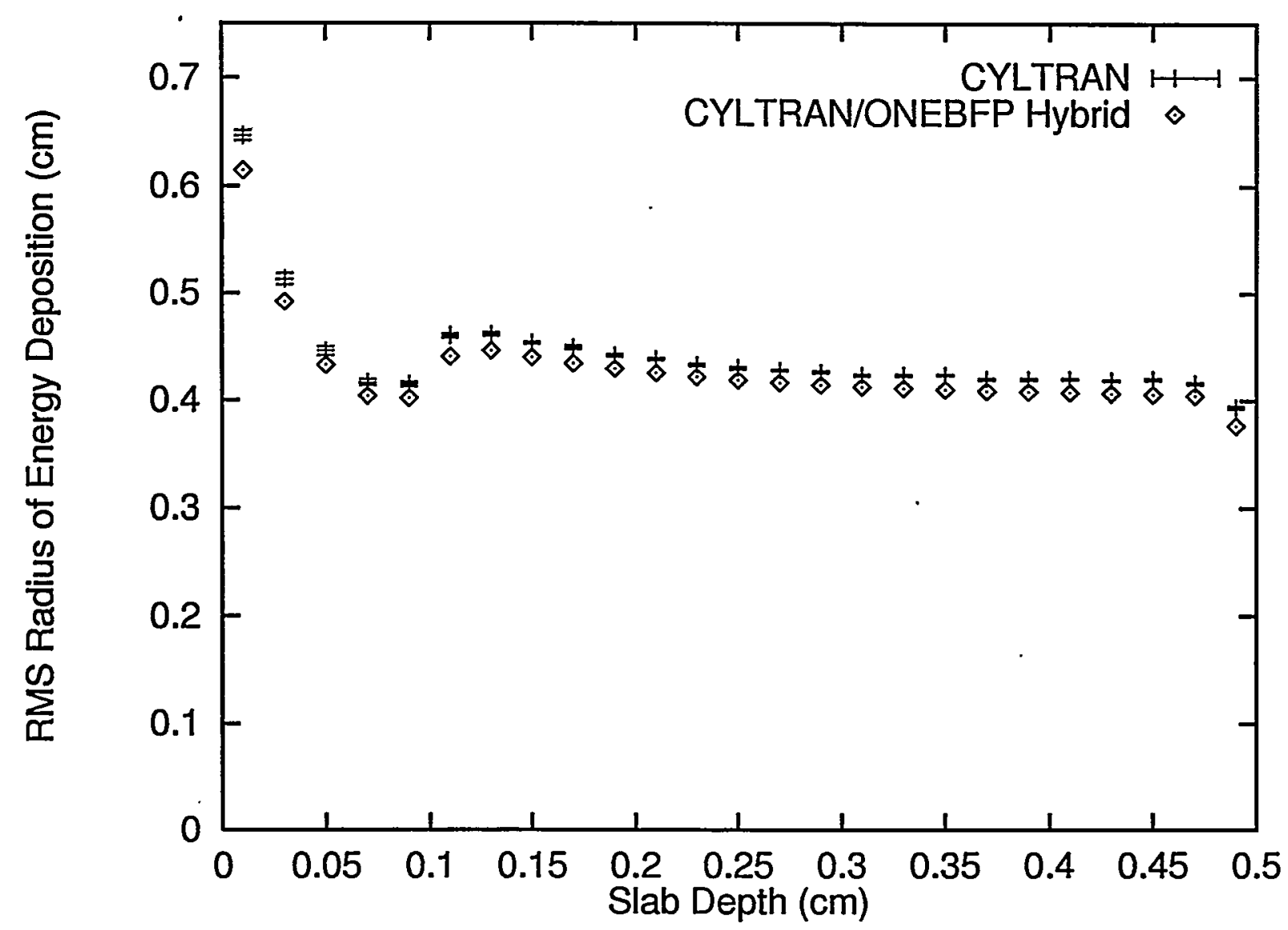

Figure 18: $\hat{r}$ versus $z$ for a $10 \mathrm{MeV}$ photon pencil beam normally incident on a slab of 0.1 $\mathrm{cm}$ copper and $0.4 \mathrm{~cm}$ phosphor. 


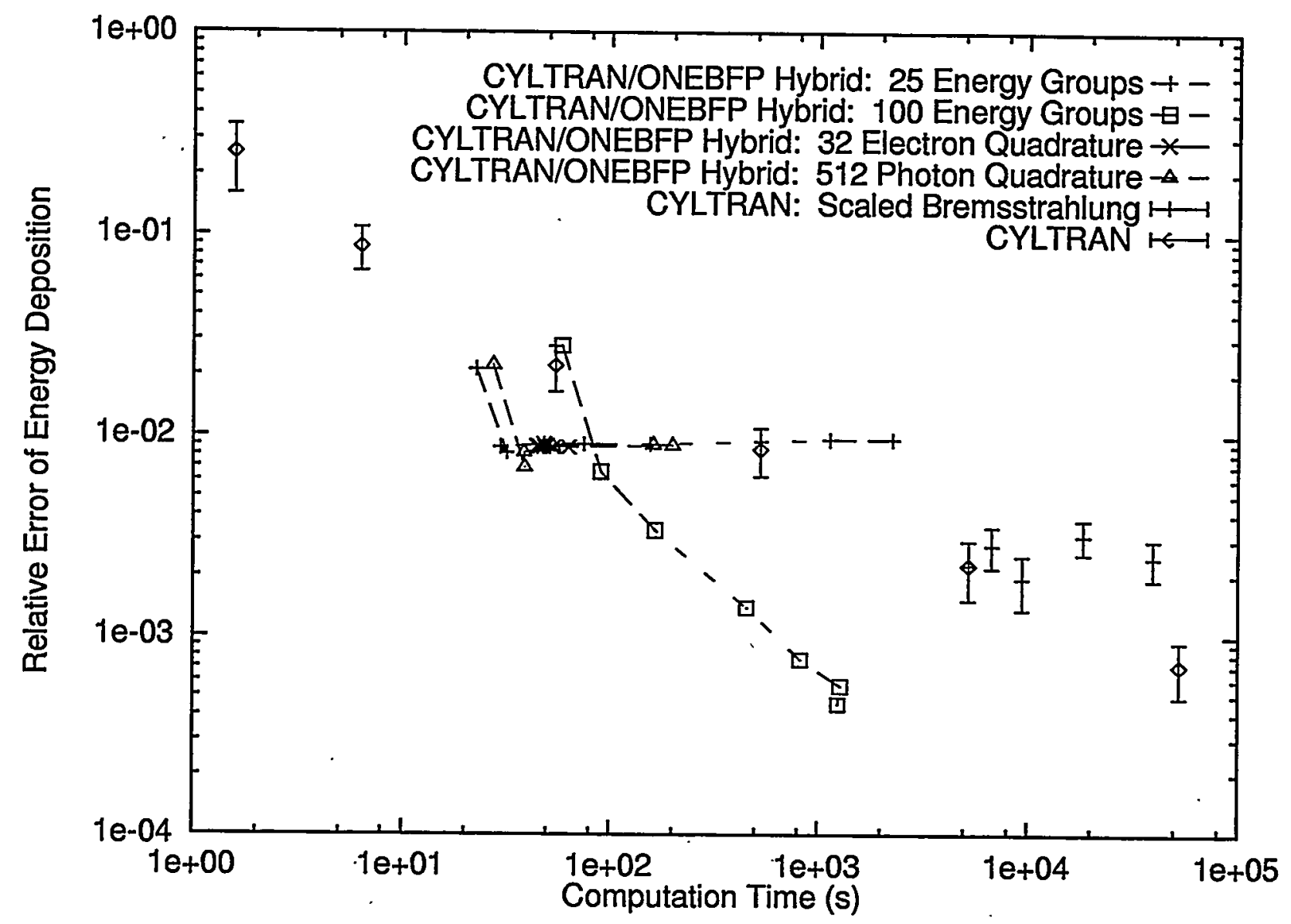

Figure 19: Relative error in $\hat{D}(z)$ versus computation time for a $10 \mathrm{MeV}$ photon pencil beam normally incident on a slab of $0.1 \mathrm{~cm}$ copper and $0.4 \mathrm{~cm}$ phosphor. 


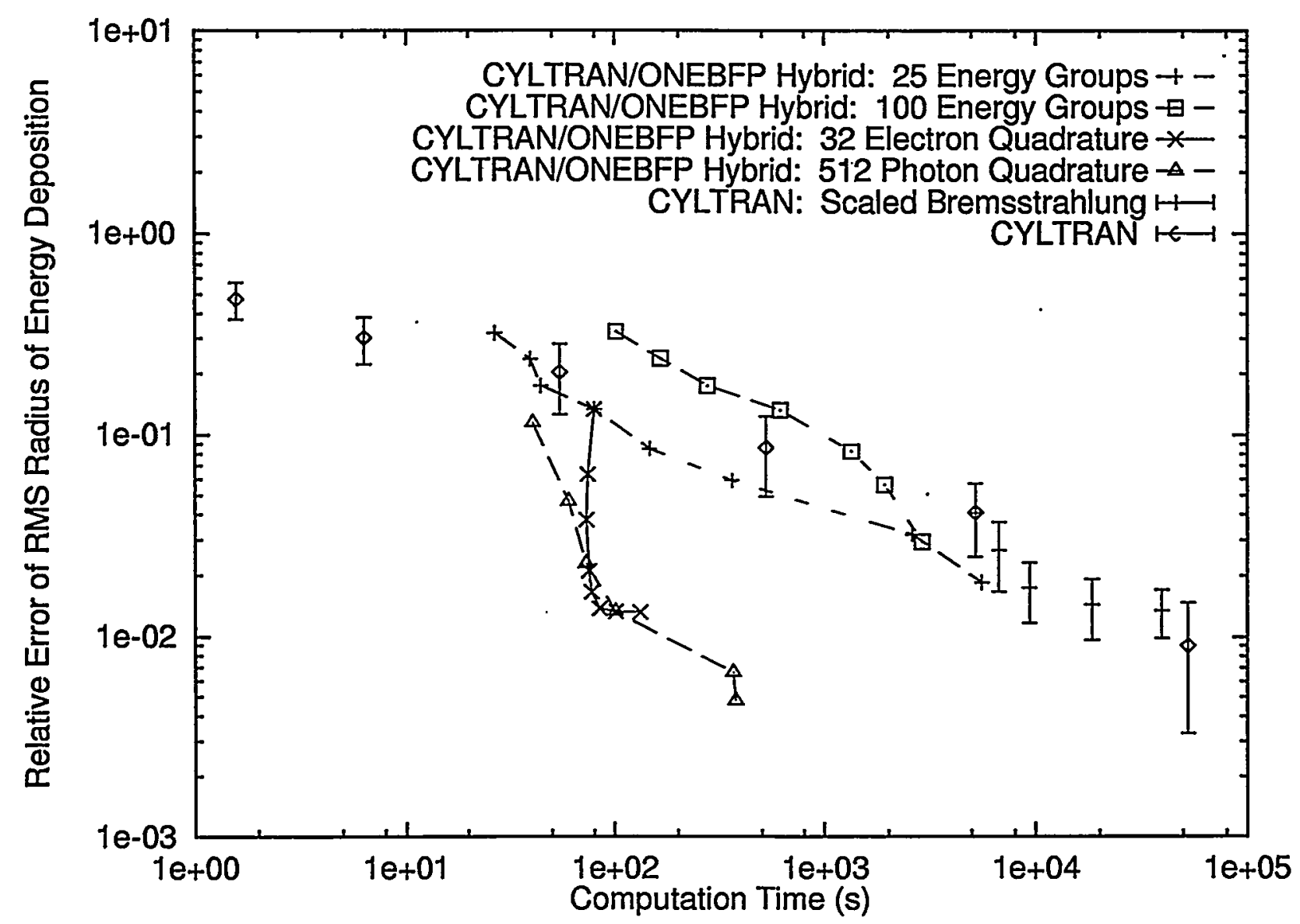

Figure 20: Relative error in $\hat{r}(z)$ versus computation time for a $10 \mathrm{MeV}$ photon pencil beam normally incident on a slab of $0.1 \mathrm{~cm}$ copper and $0.4 \mathrm{~cm}$ phosphor. 